1 (Subclinical mastitis alters dairy cow behavior and salivary SAA)

2

\title{
Changes in Behavior and Salivary Serum Amyloid A in cows with Subclinical Mastitis
}

\section{G. Caplen ${ }^{1 *}$, S.D.E. Held ${ }^{1}$}

\section{gina.caplen@bristol.ac.uk; suzanne.held@bristol.ac.uk}

${ }^{1}$ Animal Welfare and Behavior Group, Bristol Veterinary School, University of Bristol, Langford, Bristol, BS40 5DU, UK

*Corresponding author

\section{ABSTRACT}

The aim of this study was to identify detailed changes in behavior, and in salivary serum amyloid A (SAA), associated with subclinical mastitis. This included standard sickness behaviors (which form part of an adaptive response to conserve energy, minimize heat loss and facilitate recovery following infection and inflammation) and lesser-studied behaviors, that are biologically predicted to change at low-level infection, and therefore particularly relevant for detecting subclinical mastitis (here labelled 'luxury' behaviors). SAA is a physiological marker of systemic inflammation, with levels in milk and serum already known to increase during subclinical mastitis. We investigated whether the same was true for SAA in cow saliva. Data were collected for $\mathrm{n}=34$ commercial barn-housed dairy cows, tested in 17 matched-pairs. Each pair comprised a cow with subclinical mastitis (SCM) and a healthy control (CTRL), identified using somatic cell count (SCC) (SCM: SCC >200 x1000 cells/ml; CTRL: SCC $<100 \times 1000$ cells/ml). SCM cows were selected for study ad-hoc, at which point they were paired with a CTRL cow, based upon parity and calving date; consequently, the full data set was accrued over several months. Data were collected for each pair over 3 days: SCC (14:00-15:00h) Day 1; behavior (24h from 00:00h) Day 2; salivary serum amyloid-A (SAA) Day 3. We report, for the first time, that an increase in salivary SAA occurs during subclinical mastitis; SAA was higher in SCM cows and demonstrated a positive (weak) correlation with SCC. The behavioral comparisons revealed that SCM cows had reductions in activity (behavioral transitions and distance moved), social exploration, social reactivity (here: likelihood to move away/be displaced following receipt of agonism), performance of social grooming and head butts, and the receipt of agonistic non-contact challenges. In addition, SCM cows received more head swipes, and spent a greater proportion of time lying with their head on their flank than CTRL cows. SCM cows also displayed an altered feeding 
pattern; they spent a greater proportion of feeding time in direct contact with two conspecifics, and a lower proportion of feeding time at self-locking feed barriers, than CTRL cows. Behavioral measures were found to correlate, albeit loosely, with serum SAA in a direction consistent with predictions for sickness behavior. These included positive correlations with both lying duration and the receipt of all agonistic behavior, and negative correlations with feeding, drinking, the performance of all social and all agonistic behavior, and social reactivity. We conclude that changes in salivary SAA, social behavior, and activity offer potential in the detection of subclinical mastitis and recommend further investigation to substantiate and refine our findings.

Keywords: Cows; SAA; Sickness; Behavior; Subclinical mastitis

\section{INTRODUCTION}

Clinical stages of infectious disease are typically easily identified by obvious physical symptoms and behavioral changes (so-called 'sickness behaviors'; Hart, 1988). Subclinical infection, that is infection below the level of clinical detection, by definition, is more difficult to spot. However, based on the interactions between the immune and central nervous system that cause sickness behavior predictions can also be made about behavioral changes during subclinical infection (Dantzer, 2004). These behavioral changes can be used as early warning signs of disease (Weary et al. 2009; von Keyserlingk et al. 2010), and/or to identify chronic subclinical infection levels. Mastitis remains a major concern in dairy cows with serious negative effects on welfare and productivity (Petersson-Wolfe et al. 2018). At subclinical levels, inflammation is present in response to the infection and milk production drops, but no abnormalities in the gland or milk are visible (Sordillo et al., 1997). It is therefore important for infection at any level to be identified and treated as soon as possible. Our main aim was to identify such changes in cows based on a detailed behavioral comparison between individuals with spontaneously occurring subclinical mastitis and healthy controls.

Behaviors in healthy animals can be divided, on the basis of immediate survival benefits, into 'core maintenance' and 'luxury' (after e.g. Dawkins, 1990). Core behaviors have immediate, short-term survival benefits; examples include resting, feeding, and drinking. They, therefore, start to decline only at later disease stages (e.g. Littin et al., 2008) and have low sensitivity during early and low-level stages (e.g. Sepúlveda-Varas et al., 2014). In our comparison we 
68

69

70

included standard 'core' sickness behaviors, such as changes to feeding, previously shown to be associated with experimentally-induced and spontaneously-occurring clinical mastitis (Siivonen et al., 2011; Fogsgaard et al. 2012; Sepúlveda-Varas et al., 2016). Because of our focus on subclinical mastitis, we also included so-called 'luxury' behaviors, such as physical and social exploration, grooming and various social interactions. These 'luxury' behaviors have delayed, longer-term, benefits and are not essential for immediate survival, meaning that they are biologically predicted to change at lower levels of disease than other sickness behaviors due to a greater sensitivity to disease challenge (Littin et al. 2008; Weary et al. 2009), especially when energy resources are diverted to fighting infection (see also 'lowresilience behaviors' in Littin et al., 2008).

In cows, effective health monitoring is hampered by logistical difficulties associated with direct animal observations within large open barns, reductions in human interaction linked with the substantial uptake in robotic milking units, and the tendency of cattle (for several biological reasons) to display only subtle indicators of pain/weakness (Gleerup et al., 2015). Advances in image analysis now allow automated recognition of individuals within a herd (Andrew et al., 2020), and accurate identification of health-related abnormal behaviors, e.g. foot disease (Gu et al., 2017). The identification of behaviors associated with subclinical disease (prior to the development of, or without, clinical symptoms) may therefore find application in future diagnostic software algorithms targeted at early disease monitoring in dairy cows (see e.g. Wagner et al. 2020).

A second aim was to quantify levels of salivary serum amyloid A (SAA), a major acute phase protein (APP) in cows (Murata et al., 2004), with and without spontaneously occurring subclinical mastitis. APPs are non-specific inflammatory markers that fluctuate in response to infection. Increased SAA levels have been detected during subclinical mastitis within both serum and milk (Kovac et al., 2011; Kovacevi-Filipovic et al., 2012). Other studies have confirmed SAA presence in bovine saliva (Lecchi et al., 2012; Rahman et al., 2013), suggesting salivary SAA has the potential for use in non-invasive detection of infectious diseases, such as mastitis.

Raised systemic inflammation levels, whether during acute infection or as chronic inflammatory states, are predicted to be accompanied by a feeling of sickness or 'malaise' in animals, as they are in humans (Dantzer et al., 2008; Weary et al. 2009). De Boyer Des 
Roches et al. (2017) reported correlations between (serum) SAA and several pain indicators before and after experimentally induced intra-mammary challenge with E. coli, including behavioral measures of attentiveness to surroundings. This suggested a direct link between serum SAA, infection levels and some sickness behavior in mastitic cows. A non-invasive means of monitoring systemic inflammation would also benefit future investigations into the impact of spontaneously occurring infections, such as mastitis, on cow welfare. In our study we tested for associations between salivary SAA levels and SCC (a standard measure of mastitis severity), and for associations between these physiological measures and behavior.

111 In summary, the purpose of this study was to identify differences in behavior and salivary

112 SAA associated with subclinical mastitis. To this end we: (a) compared the behavior and

113 salivary SAA of cows with subclinical mastitis with that of matched healthy individuals and,

114 (b) correlated behavioral variables with both SCC and salivary SAA. We predicted that

115 salivary SAA would be higher in cows with subclinical mastitis than in matched healthy controls. We also predicted that luxury behaviors, here including a range of social behaviors, would decrease with subclinical mastitis, and that differences detected would correlate negatively with the physiological measures (SCC and salivary SAA).

\section{MATERIALS AND METHODS}

\section{$121 \quad$ 2.1. Ethics Statement}

122 The study was conducted between October 2017 and February 2018 at Bristol Veterinary

123 School dairy farm. The experimental procedures were approved by the Animal Welfare and

124 Ethical Review Board at the University of Bristol and conducted under University Investigation Number UB/17/061 'Behavioural markers of subclinical disease in dairy cows'.

\subsection{Animals}

128 Focal cows $(\mathrm{n}=34)$ were part of an indoor commercial Holstein-Friesian dairy herd $(\mathrm{n}=$ 129 200) and resided within the low milk-yield group (approx. $n=80$ cows) at the time of the 130 study; having been part of the group for at least one month prior to data collection they were well-established within the social dominance hierarchy. Low yield animals were studied as they were deemed to be of low risk for having, or developing, subclinical metritis or ketosis

133 during the trial. Cows were housed within a free-stall barn containing 93 lying cubicles $(1.2 \mathrm{x}$

$1342.4 \mathrm{~m}$ ) with sand bedding, three stainless steel tip-over drinking troughs, a swinging brush

135 (DeLaval), and automatic floor scrapers. Figure 1 shows the lay-out and relative positioning 
136 of resources within the pen. Cows were milked three times daily (at 06:00, 14:00 and 22:00h)

137 and fed a total mixed ration once daily (06:00h).

139 Only clinically healthy non-lame cows (mobility score $\leq 1$; AHDB, 2015) without physical 140 symptoms of mastitis were selected for inclusion in the study. Clinical health was assessed

141 using individual health records and visual inspection of each cow by the researcher and 142 herdsman. Data were collected concurrently from cows in matched pairs, each comprising a 143 cow with subclinical mastitis (SCM) and a clinically healthy control (CTRL). Matching was 144 performed on the basis of the following potential confounds: parity, pregnancy status (yes/no) 145 and stage of pregnancy (days since insemination). Cows with a SCC of $>200$ (x1000 cells $/ \mathrm{ml}$ ) 146 were classified as SCM (Madouasse et al., 2010), while cows with a SCC of $<100$ (x1000 147 cells $/ \mathrm{ml}$ ) were classified as CTRL. The 34 focal cows comprised 24 pregnant and 10 barren 148 ones, 20 primiparous $(n=20)$ and 14 multiparous ones (of which two cows were at their $2^{\text {nd }}$ 149 parity; two at $3^{\text {rd }}$; six at $4^{\text {th }}$; four at $\left.5^{\text {th }}\right)$. The time to expected calving date ranged from 55 150236 days. While data from the two cows in a matched pair were collected at the same time, 151 the data for 17 pairs took several months to collect due to low mastitis incidence within the 152 herd at the time of the study.

INSERT FIGURE 1 NEAR HERE

\subsection{Somatic Cell Count}

157 Composite quarter milk samples were collected between 14:00-15:00h on Day 1. Although pairs of cows had data collected at different dates, for the purpose of our experimental design, all cows had SCC data collected on Day 1 and behavioral data on Day 2. Somatic cells were manually counted using a standard direct microscopic methodology (ISO 13366-1, 1997) following staining with Newman-Lampert stain solution: Levowitz-Weber modification (Newman's Stain Solution: modified, 01375, Sigma-Aldrich).

\subsection{Behavioral Measures}

Each focal cow was fitted with a colored collar to facilitate individual recognition on Day 1. Two CCTV systems (N441L1T, Annke® , CA 91748, US), including six cameras recorded video footage from the entire low-yield pen. Continuous behavioral data were then coded retrospectively from video for each focal cow for 24h starting from 00:01h of Day 2 by a single experienced coder who was unaware of the health status of the cows at the time of 
170

171

172

173

174

175

176

177

178

179

180

181

182

183

184

185

186

187

188

189

190

191

192

193

194

195

196

197

198

199

200

201

scoring; these data made up the $24 \mathrm{~h}$ (' $\mathbf{2 4 h}$ ') data set. A second behavior data set was compiled for each cow using video recordings of the first $60 \mathrm{~min}$ following morning milking ('1hPostM1'). This hour coincided with the peak feeding time of the day, since fresh feed was delivered to the feed corridor while the cows were in the parlour at morning milking. The start time of the 60 min observation period was specific to each focal cow, starting immediately following that cow's re-entrance into the home pen from the parlour exit. All behavioural measures are described in Table 1. Three broad categories of behavior were of interest: 'core' maintenance (including lying, feeding/drinking and activity), non-social 'luxury' behaviors (such as self-grooming and exploring the physical environment), and social 'luxury' behaviors. The last category was the most detailed, comprising non-agonistic (e.g. allogrooming, social exploration) and agonistic (e.g. head swipes, head butts) behaviors, in addition to social reactivity (here defined as the likelihood of moving away/being displaced following the receipt of an agonistic behavior).

Total behavioral transitions ('Trans'), a measure of activity, was calculated using all behaviors, including those coded, but not analysed individually (i.e. not listed in Table 1). These additional non-focal behaviors included eat sand, lick salt, paw sand, run, shake head, stand, and walk. Proximity was investigated using nearest neighbour ('NN') scores. When the focal cow was located at the feed barrier or resting in a cubicle, the number of other cows in immediate 'proximity' were scored as $0 \mathrm{NN}, 1 \mathrm{NN}$ or $2 \mathrm{NN}$ (Table 1). To enable an estimation of distance moved ('Dist'), the pen floorspace was hypothetically subdivided into 29 units (each 4.8 m wide; see Figure 1) and the location of each cow was noted every five minutes throughout the $24 \mathrm{~h}$ period. The number of floor units crossed during these intervals was then used to estimate distance moved.

Data were not available for 06:00, 14:00 or 22:00h as the cows were in the milking parlour or collecting yard during these periods. To account for differences in total time visible (i.e. due to variations in time spent within the parlour) data in the ' $24 \mathrm{~h}$ ' data sets were standardised to either: number per hour visible (behavioral events) or seconds per hour visible (behavioral states); e.g. to standardise data from a cow that was visible for $20 \mathrm{~h}, 42 \mathrm{~min}$ and $12 \mathrm{~s}$ within a $24 \mathrm{~h}$ period data this data would be divided by 20.703 . 
202 Table 1: Cow behavioral measures used in the study

\begin{tabular}{|c|c|}
\hline Measure (unit) & Abbreviation - Definition \\
\hline (A.) Maintenance (Core) & \\
\hline Lie (s) & 'Lie'a: horizontal recumbent resting position, with abdomen in contact with the floor. \\
\hline Head on flank (s) & 'HOF'a: lying with the head held in contact with the flank, pointing backwards towards the rump. Associated with active sleep. \\
\hline Drink (s) & 'Drink'a: standing with muzzle placed within a water trough. \\
\hline Feed (s) & 'Feed'a: ingesting or chewing of food while standing with the head crossing the feed barrier. \\
\hline Activity: Transitions (n) & 'Trans'a: the total number of changes in behavior a focal cow undergoes during the observation period. \\
\hline Activity: Distance moved (units) & 'Dist'a: the number of units of floor space crossed by the focal cow during the observation period. \\
\hline (B.) Non-social (Luxury) & 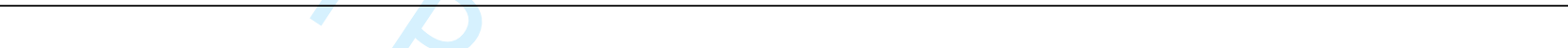 \\
\hline Brush use (s) & 'Brush'a: contact made between any body part and the mechanical brush (while standing). \\
\hline Self-groom (s) & $\begin{array}{l}\text { 'Self-groom'a }=\text { 'Lick self' (lick own body part) + 'Rub self' (rub own body part against pen furniture) + 'Scratch self' (using own } \\
\text { foot to scratch own body part). }\end{array}$ \\
\hline Explore environment (s) & $\begin{array}{l}\text { 'ExpEnv'a = 'Explore food' (holding nose close to food while sniffing or moving/flicking nose around within food, without any } \\
\text { obvious active ingestion) + 'Explore pen' (licking or holding nose very close to any part of the barn structure or pen furniture) + } \\
\text { 'Explore sand' (holding nose close to sand bedding or flicking sand with nose). }\end{array}$ \\
\hline $\begin{array}{l}\text { (C.1) Social (Luxury): } \\
\text { agonistic interactions and } \\
\text { displacement }\end{array}$ & $\begin{array}{l}\text { Each behavior was further classified as: given }(\mathrm{G}) \text {, received }(\mathrm{R}) \text {, or received with displacement (+D) (i.e. recipient moves away/ is } \\
\text { displaced) see e.g. 'Body push'. }\end{array}$ \\
\hline Body push: given (n) & $\begin{array}{l}\text { 'BPG'a: use of sudden impact contact made with the flank delivering a sideways shunt to the head or flank of a recipient; frequently } \\
\text { results in displacement (e.g. when accessing a crowded feed barrier). }\end{array}$ \\
\hline Body push: received (n) & 'BPR'a: receipt of a body push from another cow \\
\hline Body push: displacement (\%) & $\begin{array}{l}\text { 'BPR+D': receipt of a body push, immediately ( } \leq 2 \mathrm{~s}) \text { prompting the focal cow to be displaced }) \text {. } \\
\text { '\%oBPR+D'b }=\left({ }^{\prime} B P R+D ' /{ }^{\prime} B P R '\right) \text { x } 100\end{array}$ \\
\hline Non-contact challenge & 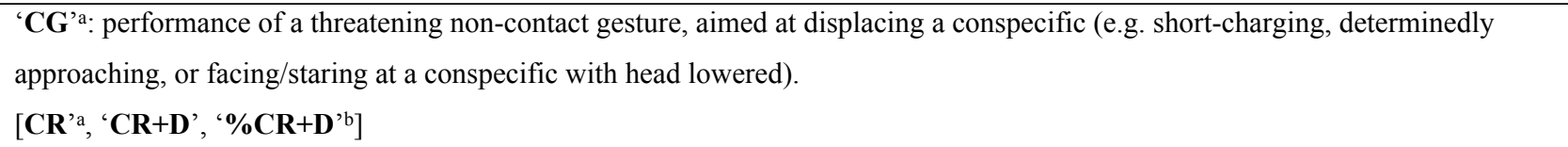 \\
\hline
\end{tabular}




\begin{tabular}{|c|c|}
\hline Head butt & 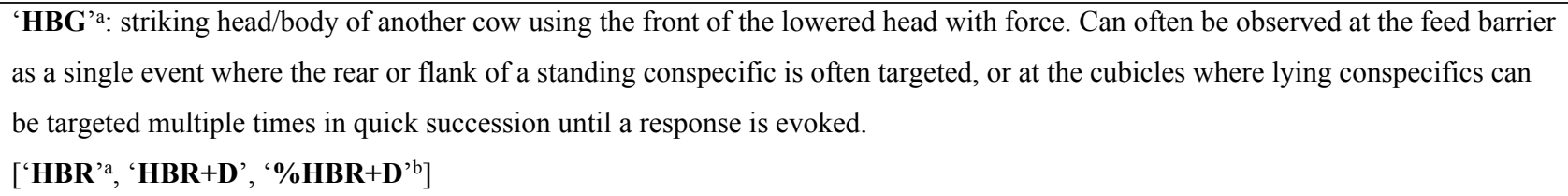 \\
\hline Head push & $\begin{array}{l}\text { 'HPG'a: the use of prolonged/sustained contact of the forehead (occasionally the lateral aspect of the head) applied to a conspecifics } \\
\text { head or body. } \\
{\left[{ }^{[} \mathbf{H P R}{ }^{\prime a},{ }^{\prime} \mathbf{H P R}+\mathbf{D}^{\prime},{ }^{\prime} \% \mathbf{H P R}+\mathbf{D}^{\prime b}\right]}\end{array}$ \\
\hline Head swipe & 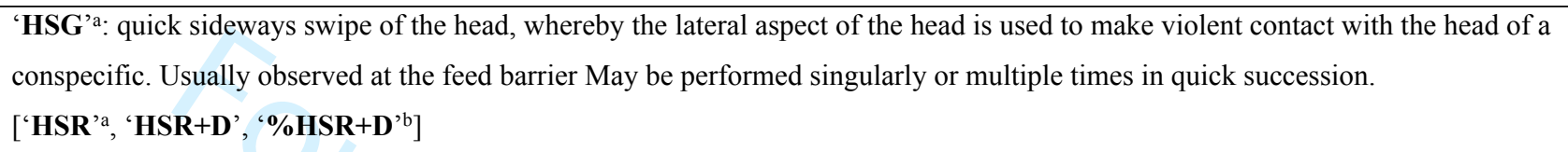 \\
\hline Total agonistic: given (n) & 'FocSocG'a $=$ 'BPG' + 'CG' +'HBG' + 'HPG' + 'HSG' \\
\hline Total agonistic: received (n) & 'FocSocR'a = 'BPR'+ 'CR'+ 'HBR' + 'HPR' + 'HSR' \\
\hline $\begin{array}{l}\text { ‘All social reactivity' = percentage } \\
\text { displacement following all agonistic } \\
\text { interactions received }\end{array}$ & 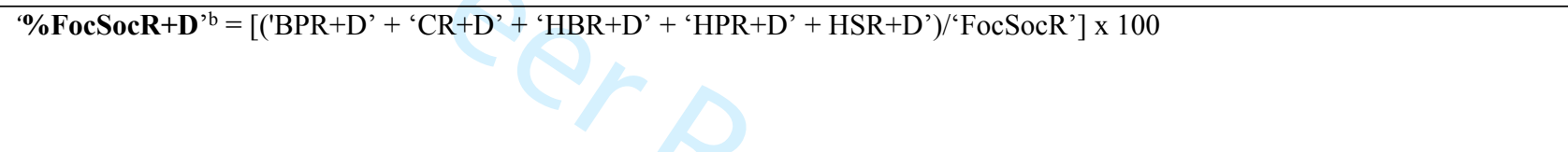 \\
\hline Mutual head butt (n/s) & $\begin{array}{l}\text { "MutHdButt'a: mutual head-to-head butting between the focal cow and conspecifc. Usually observed in prolonged bouts whereby } \\
\text { the two cows stand head-down facing each other with/without sustained forehead contact between each butt. }\end{array}$ \\
\hline $\begin{array}{ll}\text { (C.2) } & \text { Social (Luxury): } \\
& \text { non-agonistic }\end{array}$ & \\
\hline Allo-groom give $(\mathrm{n} / \mathrm{s})$ & 'AlloG'a: licking any body part of a conspecific (most frequently the head or main torso). Usually observed in prolonged bouts. \\
\hline Allo-groom receive $(\mathrm{n} / \mathrm{s})$ & 'AlloR'a: receipt of licks from a conspecific. During licking the recipient usually stops all other activity and stands still. \\
\hline Explore social (s) & $\begin{array}{l}\text { 'ExpSoc' }{ }^{\prime a}=\text { 'Explore cow' (nose positioned close to another cow in the act of sniffing - no reciprocation) + 'Mutual sniff' } \\
\text { (reciprocal sniffing between a focal cow and conspecific, usually stood facing one-another with noses almost touching). }\end{array}$ \\
\hline Mutual head rub (n/s) & 'MutHdRub'a: mutual head-to-head rubbing performed by a focal cow and a conspecific. Usually observed in bouts. \\
\hline \multicolumn{2}{|l|}{ (C.3) Total social interactions } \\
\hline Total social interactions: given (n) & $\begin{array}{l}\text { 'SocG'a = 'AlloG' + 'BPG' + 'CG' + 'Chin rest give' (use of chin to exert pressure on the lateral posterior of a conspecific) + } \\
\text { 'HBG' + 'HPG' + 'Head rub give' (rubbing head on conspecific without reciprocation) + 'HSG' + 'Mount give' (bulling behavior } \\
\text { where focal cow stands on back legs and rests her chest on the back/rump of a conspecifc) + 'MutHdButt' + 'MutHdRub' }\end{array}$ \\
\hline
\end{tabular}




\begin{tabular}{|c|c|}
\hline Total social interactions: received (n) & $\begin{array}{l}\text { 'SocR' }{ }^{\prime a}=\text { 'AlloR' + 'BPR' + 'CR' + 'Chin rest receive’ + 'HBR’ + 'HPR' + 'Head rub receive’ + 'HSR' + 'Mount receive’ + } \\
\text { 'MutHdButt' + 'MutHdRub' }\end{array}$ \\
\hline $\begin{array}{l}\text { (D.) Social Proximity and feed } \\
\text { barrier preference }\end{array}$ & \\
\hline Feed barrier: all (s) & 'FB_All': total time spent with the head positioned over the feed barrier regardless of type (i.e. open-rail or self-locking). \\
\hline Feed barrier: no near neighbours (\%) & $\begin{array}{l}\text { 'FB_0NN': time spent with the head positioned over the feed barrier without any direct flank-to-flank contact with conspecifics. } \\
\text { '\%FB_ONN'b }=\left({ }^{\circ} \mathrm{FB} \_0 \mathrm{NN} \text { ' } / \text { 'FB_All' }\right) \times 100\end{array}$ \\
\hline Feed barrier: two near neighbours (\%) & $\begin{array}{l}\text { 'FB_2NN': time spent with the head positioned over the feed barrier with direct flank-to-flank contact with two other cows (i.e. } \\
\text { cows positioned on either side). } \\
\text { '\%FB_2NN'b }=\text { ('FB_2NN'/FB_All') x } 100\end{array}$ \\
\hline Lie: no near neighbours (\%) & 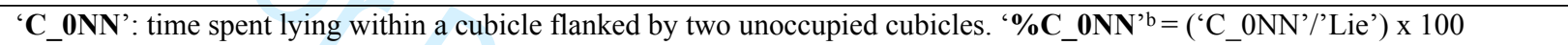 \\
\hline Lie: two near neighbours (\%) & 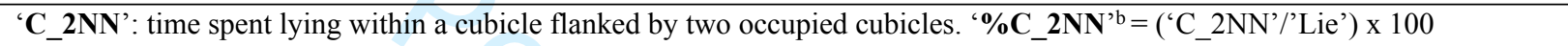 \\
\hline Feed barrier: open (\%) & $\begin{array}{l}\text { 'FB_Open': time spent with the head positioned over the open-rail section of the feed barrier. } \\
\text { '\%FB_Open'b= ('FB_Open'/'FB_All') x } 100\end{array}$ \\
\hline
\end{tabular}

a inclusion in 24h and 1hPostM1 data sets, ${ }^{\mathrm{b}}$ inclusion in 24h data set only 


\subsection{Saliva Collection and SAA}

Saliva was collected (Day 3) using a cotton swab (SalivaBio Children's Swab, Item No. 5001.06, Salimetrics) and then immediately stored at $-80^{\circ} \mathrm{C}$ prior to analysis. SAA was measured in saliva from 31 cows (saliva volumes from three cows were too small to be analysed), diluted 1:2, using a commercially available kit (Bovine Serum amyloid A protein ELISA Kit, EB0015, Finetest ${ }^{\circledR}$, Wuhan Fine Biotech Co. Ltd.). To assess the suitability of the kit for use with saliva an assay validation was performed. To determine parallelism (linearity) a displacement curve, produced by double-diluting a pooled saliva sample with assay buffer, was compared to a standard curve. Percentage binding (as a percentage of that recorded for the zero standard) was calculated, in addition to the Log of the standard concentration (SAA standard) and the Log of the inverse of the dilution factor (saliva sample), e.g. 1:4 was transformed to $\log (1 / 4)$. Parallelism was confirmed using a statistical test for the analysis of covariance (ANCOVA, SPSS). To measure assay accuracy the percentage recovery of exogenous SAA was calculated following the addition of $300 \mathrm{ng} / \mathrm{ml}$ SAA standard to a pooled saliva sample. Precision was assessed via intra- and inter-assay coefficients of variation $(\mathbf{C V})$; the former was determined following the repeated measurement of aliquots of pooled saliva containing either high (quality control: $\left.\mathbf{Q} \mathbf{C}_{\mathbf{h i g h}}\right)$ or low $\left(\mathbf{Q C}_{\text {low }}\right)$ endogenous SAA within the same plate, while the later was determined following the assay of $\mathrm{QC}_{\mathrm{high}}$ and $\mathrm{QC}_{\text {low }}$ samples in different plates.

\subsection{Statistics}

Following tests for normality (Shapiro-Wilk analysis), all behavioral measures, SAA and SCC were compared between CTRL and SCM using Paired samples t-test or Wilcoxon Signed-Rank tests (SPSS Statistics 24.0). Behavioral data from the continuous $24 \mathrm{~h}$ data set and the data sub-set (60 min after the morning milking, '1hPostM1') were separately analysed. Since the experimental design required the performance of multiple comparisons between measures there was an increased associated risk of Type I errors. Use of Bonferroni correction procedures has been highlighted as problematic (especially for animal behavioral studies, where sample sizes are often small) due to their tendency to increase Type II errors (Nakagawa, 2004). As an alternative to standard correction procedures we, therefore, calculated measures of observed (standardised) effect size in addition to p-values. Effect size measures the strength/magnitude of a relationship and, thereby, helps us to determine the strength of a statistical claim and whether a difference is real (i.e. it enables us to judge biological importance). Hedges' g-value (Equations 1 and 2), also termed 'Cohen's d-value 
239 for paired samples' (Hedges, 1981; Cohen, 1988; Nakagawa and Cuthill, 2007) and 95\%

240 confidence intervals (CI) for effect size (Equations 3 and 4), were calculated for all measures

241 that met the assumptions of normality.

$$
g=\frac{\bar{x}_{2}-\bar{x}_{1}}{\sigma_{\text {paired }}}
$$

243 where

$$
\sigma_{\text {paired }}=\sqrt{\frac{\left(n_{2}-1\right) s_{2}^{2}+\left(n_{1}-1\right) s_{1}^{2}}{n_{1}+n_{2}-2}}
$$

$\overline{x_{1}}$ and $\overline{x_{2}}$ are the means of the two groups, $\sigma_{\text {paired }}$ is the pooled standard deviation, $n$ is the number of data points, and $s^{2}$ is the sample variance.

$$
95 \% \mathrm{CI}=g-1.96 s e_{\mathrm{g}} \text { to } g+1.96 s e_{\mathrm{g}}
$$

250 where

$$
s e_{\mathrm{g}}=\sqrt{\frac{2\left(1-r_{1,2}\right)}{n}+\frac{g^{2}}{2(n-1)}}
$$

252

253

254

255

256

257

258

259

260

261

262

263

264

$s e_{\mathrm{g}}$ is the asymptotic standard error for the effect size, $n=n_{1}=n_{2}$, and $r_{1,2}$ is the correlation coefficient between the two groups. For all behavioral measures that did not meet the assumptions of normality, bootstrap effect size values (Hedges' g-value with 95\%CI, R = 2000) were computed using the software package 'bootES' (Gerlanc and Kirby, 2012; Kirby and Gerlanc, 2013) and R (Version 3.2.2., www.r-project.org/). Effect size statistics were interpreted as follows: (a) the size of the effect (based upon the estimated g-values: $\leq 0.39=$ small, $0.40-0.79=$ medium, $\geq 0.80=$ large); (b) statistical significance (attributed to all measures where the associated 95\%CI did not contain ' 0 ') (Lee, 2016).

Interpretation of statistically non-significant $\mathrm{p}$-values is possible using effect size confidence intervals in combination with the effect size (see Nakagawa \& Foster, 2004). To identify those measures that were not statistically significantly different between SCM and CTL cows in the continuous $24 \mathrm{~h}$ data set but could yet be biologically important, we used information from published studies to set broad, accepted, relative difference levels (RDL\%, Table 2). Although these studies are not specific to subclinical mastitis, examples include the impact of clinical levels of (non-mastitic) infection and social status on behavior, it is assumed that they provide generous and relevant difference levels with which to compare our subclinical findings. 
272 Table 2: Summary of the relative difference levels (RDL\%) and their sources, used in this study to 273 ascertain the existence of a biologically meaningful difference in behavioral measures between cows, 274 with and without subclinical mastitis, based on effect size

\begin{tabular}{|l|c|l|}
\hline Behavioral Measure $^{1}$ & RDL\% & Reference \\
\hline Agonistic social given: all categories & 10 & Sepulveda-Vares et al., 2016 \\
\hline $\begin{array}{l}\text { Agonistic social received: all } \\
\text { categories }\end{array}$ & 10 & Neave et al., 2018 \\
\hline \%FB_0NN, \%FB_2NN & 20 & Manson and Appleby, 1990 \\
\hline \%FB_Open & 10 & Huzzey et al., 2006 \\
\hline AlloG & 30 & Galindo and Broom, 2002 \\
\hline AlloR & 80 & Galindo and Broom, 2002; Hoonhout et al., 2017 \\
\hline Brush & 30 & Mandel et al., 2017 \\
\hline Self-groom & 20 & Fogsgaard et al., 2012 \\
\hline Activity: Trans, Dist & 10 & King et al., 2018; Steensels et al., 2017 \\
\hline Feed & 10 & Dollinger and Kaufmann, 2013 \\
\hline Drink & 20 & Huzzey et al., 2007 \\
\hline Lie & 10 & Toaff Rosenstein et al., 2016 \\
\hline Other & 20 & \\
\hline
\end{tabular}

1\% FB_0NN = percentage of time at feed barrier with no near neighbors; \%FB_2NN = percentage of time at feed barrier with two near neighbors; \%FB_Open = percentage of time at open-rail feed barrier; AlloG = allogroom give; AlloR = allogroom receive; Brush = use of revolving brush; Self-groom = licking, rubbing, scratching self; Trans $=$ behavioral transitions; Dist $=$ distance moved; Lie $=$ lying in cubicle.

For those measures where no relevant literature was available ('Other'; Table 2), we used the average of the known RDLs (i.e. 20\%; Table 2). For each measure, relative difference values (RDV) were then calculated using the RDL\% and the respective mean value from the CTRL group. $95 \% \mathrm{CI}_{\mathrm{RDV}}$ were calculated using the confidence intervals from the effect size statistics and the (between-group) difference in means. In those cases where the $95 \% \mathrm{CI}_{\mathrm{RDV}}$ did not include the RDV, we conclude (with 95\% confidence) that the current study showed no important biological effect for that measure; we refer to these as 'biologically unimportant'. In cases where the $95 \% \mathrm{CI}_{\mathrm{RDV}}$ did include the $\mathrm{RDV}$, we conclude that a difference was inconclusive but plausible; we refer to these as 'biologically inconclusive'. For example, if CTRL cows performed more body pushes than the SCM cows, yet this difference failed to reach statistical significance $(\mathrm{P} \geq 0.10)$, using the $\mathrm{p}$-value alone we would dismiss this behavior as being unaffected by subclinical inflammation. However, if the RDV for this behavior was within the $95 \% \mathrm{CI}_{\mathrm{RDV}}$ range (e.g. $\mathrm{RDV}=0.08,95 \% \mathrm{CI}_{\mathrm{RDV}}=-0.08$ to 0.27 ) we would conclude that, although this effect is biologically inconclusive based upon our 
294 evidence, the difference may become significant given a larger sample size. Alternatively, if

295 the RDV, in the above example, was 0.3 , then we would conclude that the effect was

296 biologically unimportant.

297

298 To test for correlations between physiological (SCC and SAA) and behavioral measures, we

299 performed curve estimation regression statistics using the continuous $24 \mathrm{~h}$ data set (SPSS:

300 ANOVA, coefficient of determination) following tests for normality. Due to the small sample

301 size, standard deviations for the behavioral measures were already large, so outliers $( \pm 2 \mathrm{SD})$

302 deemed to be atypically/excessively low or high were considered and removed prior to data

303 analysis. Such outliers comprised a maximum of a single data point (i.e. one matched pair)

304 for any measure.

305

306

\section{RESULTS}

3.1. Behavioral Differences over 24 hours (24h data set), and during $60 \mathrm{~min}$ immediately following first milking (1hPostM1 data subset)

309

\subsubsection{Luxury behavior: Social}

$311 \quad$ (a) Total social interactions

312 In the 24h data, no significant differences were found in the total performance or receipt of

313 social interactions(Table 3). The difference in the total performance of social interactions was

314 classified as biologically unimportant in the context of this dataset ('SocG': RDV $=0.50$,

$31595 \% \mathrm{CI}_{\mathrm{RDV}}=-0.26$ to 0.43 ). Total receipt of social interactions, however, was biologically

316 inconclusive on the basis of RDV confidence intervals ('SocR': $\mathrm{RDV}=0.44,95 \% \mathrm{CI}_{\mathrm{RDV}}=$ -

3170.21 to 0.64 ). This means that given a larger sample size, significant differences might

318 become apparent in the latter measure; i.e. it may be possible to confirm that SCM cows do

319 receive fewer social interactions. Indeed, during the 60 minutes following morning milking,

320 SCM cows did receive significantly fewer social interactions (1hPostM1; Table 4).

(b) Agonistic interactions

323 Broken down into separate interaction types, SCM cows performed significantly fewer head butts ('HBG': 24h; 1hPostM1) and head pushes ('HPG': 1hPostM1) than healthy controls (Tables 3 and 4). Non-significant measures of agonistic interactions given over $24 \mathrm{~h}$ were classified as biologically inconclusive on the basis of RDV confidence intervals, again suggesting a larger sample might confirm an effect. These included body pushes ('BPG': 
$\mathrm{RDV}=0.08,95 \% \mathrm{CI}_{\mathrm{RDV}}=-0.08$ to 0.27$)$; non-contact challenges ( ${ }^{\prime} \mathbf{C G}$ ': $\mathrm{RDV}=0.02$, $95 \% \mathrm{CI}_{\mathrm{RDV}}=0.00$ to 0.15 ), head pushes ('HPG': $\mathrm{RDV}=0.03,95 \% \mathrm{CI}_{\mathrm{RDV}}=-0.03$ to 0.18 ), and head swipes ('HSG': $\mathrm{RDV}=0.17,95 \% \mathrm{CI}_{\mathrm{RDV}}=-0.12$ to 0.63 ) as well as total agonistic interactions ('FocSocG': $\mathrm{RDV}=0.40,95 \% \mathrm{CI}_{\mathrm{RDV}}=-0.31$ to 0.73 ).

Over the $24 \mathrm{~h}$ period, SCM cows received significantly fewer non-contact challenges than healthy cows, but more head swipes (Table 3). With the exception of body push received, which was classified as biologically unimportant ('BPR': $\mathrm{RDV}=0.08,95 \% \mathrm{CI}_{\mathrm{RDV}}=-0.01$ 0.02), all non-significant measures of agonistic interactions received over $24 \mathrm{~h}$ were classified as biologically inconclusive on the basis of RDV confidence intervals, indicating that a larger sample might confirm an effect. This included head butts ('HBR': $\mathrm{RDV}=0.07,95 \% \mathrm{CI}_{\mathrm{RDV}}=$ -0.05 to 0.13 ), head pushes ('HPR': $\mathrm{RDV}=0.02,95 \% \mathrm{CI}_{\mathrm{RDV}}=-0.00$ to 0.14 ) and mutual butting ('MutHdButt': RDV $=1.13 \mathrm{~s}, 95 \% \mathrm{CI}_{\mathrm{RDV}}=-0.19$ to $8.03 \mathrm{~s}$ ), as well as total agonistic interactions ('FocSocR': RDV $=0.34,95 \% \mathrm{CI}_{\mathrm{RDV}}=-0.13$ to 0.40 ).

During the 60 minutes following morning milking cows with subclinical mastitis received significantly fewer total agonistic interactions than healthy controls, and specifically fewer body pushes and head butts (1hPostM1; Table 4).

Table 3: Measures of social and other 'luxury' behavior recorded from pair-matched cows with (SCM) or without (CTRL) sub-clinical mastitis over 24h: parametric data $(\mathrm{P})$ analysed using paired t-test, nonparametric data (NP) analysed using Wilcoxon SR test. All units (except \%) transformed to 'per hour visible'.

\begin{tabular}{|l|c|c|c|c|c|c|c|c|}
\hline \multirow{2}{*}{$\begin{array}{l}\text { Measure } \\
\text { (unit) }\end{array}$} & \multirow{2}{*}{ Test } & \multicolumn{2}{|c|}{ CTRL } & \multicolumn{2}{c|}{ SCM } & \multicolumn{3}{c|}{ Effect Size Statistics } \\
\cline { 3 - 8 } & & Mean & SD & Mean & SD & $\begin{array}{c}\text { Hedges' } \\
\text { g }\end{array}$ & 95\%CI & $\begin{array}{c}\text { Size of } \\
\text { effect }^{2}\end{array}$ \\
\hline SocG $^{\ddagger},(\mathrm{n})$ & $\mathrm{NP}$ & $5.0^{\mathrm{a}}$ & 2.5 & $4.5^{\mathrm{a}}$ & 2.2 & 0.20 & $-0.53-0.87$ & Small, ns \\
\hline SocR $^{\ddagger},(\mathrm{n})$ & $\mathrm{P}$ & $4.4^{\mathrm{a}}$ & 1.1 & $5.0^{\mathrm{a}}$ & 2.5 & -0.34 & $-0.34-1.02$ & Small, ns \\
\hline FocSocG $^{\dagger},(\mathrm{n})$ & $\mathrm{P}$ & $4.0^{\mathrm{a}}$ & 2.6 & $3.3^{\mathrm{a}}$ & 1.5 & 0.32 & $-0.48-1.12$ & Small, ns \\
\hline FocSocR $^{\ddagger},(\mathrm{n})$ & $\mathrm{P}$ & $3.4^{\mathrm{a}}$ & 1.2 & $3.9^{\mathrm{a}}$ & 1.8 & -0.30 & $-0.30-0.90$ & Small, ns \\
\hline BPG $^{\ddagger},(\mathrm{n})$ & $\mathrm{NP}$ & $0.8^{\mathrm{a}}$ & 0.8 & $0.5^{\mathrm{a}}$ & 0.3 & 0.47 & $-0.25-0.90$ & Med, ns \\
\hline BPR $^{\dagger},(\mathrm{n})$ & $\mathrm{P}$ & $0.8^{\mathrm{a}}$ & 0.3 & $0.8^{\mathrm{a}}$ & 0.4 & 0.11 & $-0.31-0.53$ & Small, ns \\
\hline $\mathbf{C G}^{\ddagger},(\mathrm{n})$ & $\mathrm{P}$ & $0.2^{\mathrm{a}}$ & 0.2 & $0.1^{\mathrm{b}}$ & 0.1 & 0.62 & $-0.01-1.25$ & Med, ns \\
\hline $\mathbf{C R}^{\ddagger},(\mathrm{n})$ & $\mathrm{P}$ & $0.3^{\mathrm{a}}$ & 0.3 & $0.2^{\mathrm{c}}$ & 0.1 & 0.84 & $0.02-1.66$ & Large, sig \\
\hline
\end{tabular}




\begin{tabular}{|c|c|c|c|c|c|c|c|c|}
\hline HBG $^{\ddagger}$, (n) & $\mathrm{P}$ & $1.1^{\mathrm{a}}$ & 0.9 & $0.6^{\mathrm{b}}$ & 0.4 & 0.65 & $0.10-1.20$ & Med, sig \\
\hline HBR $^{\ddagger}$, (n) & $\mathrm{P}$ & $0.7^{\mathrm{a}}$ & 0.4 & $0.6^{\mathrm{a}}$ & 0.3 & 0.37 & $-0.45-1.19$ & Small, ns \\
\hline HPG*, (n) & $\mathrm{NP}$ & $0.3^{\mathrm{a}}$ & 0.4 & $0.1^{\mathrm{a}}$ & 0.1 & 0.52 & $-0.15-1.04$ & Med, ns \\
\hline HPR , (n) & $\mathrm{P}$ & $0.2^{\mathrm{a}}$ & 0.2 & $0.1^{\mathrm{b}}$ & 0.2 & 0.64 & $-0.01-1.29$ & Med, ns \\
\hline HSG $^{\dagger},(\mathrm{n})$ & $\mathrm{P}$ & $1.7^{\mathrm{a}}$ & 0.8 & $2.3^{\mathrm{a}}$ & 1.4 & -0.47 & $-0.23-1.18$ & Med, ns \\
\hline $\mathbf{H S R}^{\ddagger},(\mathrm{n})$ & $\mathrm{P}$ & $1.4^{\mathrm{a}}$ & 0.9 & $2.0^{\mathrm{b}}$ & 1.5 & -0.51 & $0.01-1.02$ & Med, sig \\
\hline MutHdButt ${ }^{\ddagger}$, (s) & NP & $11.3^{\mathrm{a}}$ & 11.9 & $5.3^{\mathrm{a}}$ & 4.0 & 0.66 & $-0.03-1.34$ & Med, ns \\
\hline \%FocSocR+D ${ }^{\dagger},(\%)$ & $\mathrm{NP}$ & $49.1^{\mathrm{a}}$ & 11.1 & $39.2^{\mathrm{d}}$ & 11.6 & 0.87 & $0.11-1.63$ & Large, sig \\
\hline \%BPR+D & NP & $51.5^{\mathrm{a}}$ & 14.0 & $53.7^{\mathrm{a}}$ & 17.9 & -0.11 & $-0.53-0.85$ & Small, ns \\
\hline$\% \mathrm{CR}+\mathrm{D} *,(\%)$ & $\mathrm{NP}$ & $84.4^{\mathrm{a}}$ & 13.4 & $76.9^{a}$ & 23.3 & 0.39 & $-0.32-1.10$ & Small, ns \\
\hline$\%$ \%BR+D”, (\%) & $\mathrm{P}$ & $69.9^{\mathrm{a}}$ & 15.5 & $52.7^{\mathrm{d}}$ & 16.8 & 1.10 & $0.46-1.74$ & Large, sig \\
\hline \%HPR+D ${ }^{\S},(\%)$ & $\mathrm{NP}$ & $52.8^{\mathrm{a}}$ & 25.5 & $22.3^{\mathrm{c}}$ & 35.0 & 0.96 & $-0.02-2.23$ & Large, ns \\
\hline$\% H S R+D \ddagger(\%)$ & $\mathrm{P}$ & $32.9^{\mathrm{a}}$ & 13.3 & $24.7^{\mathrm{c}}$ & 13.6 & 0.63 & $0.03-1.22$ & Med, sig \\
\hline $\operatorname{ExpSoc}^{\star}$, (s) & $\mathrm{P}$ & $18.7^{\mathrm{a}}$ & 12.3 & $9.1^{\mathrm{d}}$ & 5.4 & 1.04 & $0.30-1.78$ & Large, sig \\
\hline \%FB_0NN ${ }^{\dagger},(\%)$ & $\mathrm{P}$ & $41.4^{\mathrm{a}}$ & 12.9 & $36.0^{\mathrm{b}}$ & 13.0 & 0.43 & $-0.02-0.88$ & Med, ns \\
\hline \%FB_2NN $*$, (\%) & $\mathrm{P}$ & $24.6^{\mathrm{a}}$ & 9.7 & $30.0^{\mathrm{c}}$ & 9.8 & -0.56 & $0.11-1.01$ & Med, sig \\
\hline \%FB_Open ${ }^{\dagger},(\%)$ & $\mathrm{P}$ & $82.2^{\mathrm{a}}$ & 12.0 & $90.1^{\mathrm{c}}$ & 7.7 & -0.81 & $0.10-1.53$ & Large, sig \\
\hline \%C_ONN ${ }^{\dagger},(\%)$ & $\mathrm{P}$ & $38.8^{\mathrm{a}}$ & 20.3 & $35.8^{\mathrm{a}}$ & 20.7 & 0.43 & $-0.02-0.88$ & Med, ns \\
\hline$\% \mathbf{O C}_{2} \mathbf{N N} \mathbf{N}^{\dagger},(\%)$ & $\mathrm{NP}$ & $21.9^{\mathrm{a}}$ & 19.6 & $18.6^{\mathrm{a}}$ & 20.6 & 0.08 & $-0.81-0.58$ & Small, ns \\
\hline AlloG & $\mathrm{P}$ & $18.2^{\mathrm{a}}$ & 14.0 & $10.1^{\mathrm{c}}$ & 9.7 & 0.70 & $0.05-1.35$ & Med, sig \\
\hline AlloR $\$$, (s) & $\mathrm{NP}$ & $13.8^{\mathrm{a}}$ & 11.4 & $15.9^{\mathrm{a}}$ & 14.7 & -0.16 & $-0.54-0.79$ & Small, ns \\
\hline Brush $^{\ddagger},(\mathrm{s})$ & $\mathrm{NP}$ & $29.0^{\mathrm{a}}$ & 43.9 & $11.9^{\mathrm{a}}$ & 9.0 & 0.53 & $-0.16-0.96$ & Med, ns \\
\hline Self-groom ${ }^{\ddagger},(\mathrm{s})$ & $\mathrm{P}$ & $21.2^{\mathrm{a}}$ & 7.9 & $26.0^{\mathrm{a}}$ & 10.8 & -0.27 & $-0.58-1.12$ & Small, ns \\
\hline ExpEnv $v^{\ddagger},(\mathrm{s})$ & $\mathrm{P}$ & $55.4^{\mathrm{a}}$ & 20.9 & $50.0^{\mathrm{a}}$ & 14.5 & 0.31 & $-0.24-0.86$ & Small, ns \\
\hline
\end{tabular}

${ }^{a-d}$ Mean values in the same row with different superscripts differ as follows: ${ }^{b} p<0.10$ (trend); ${ }^{c} p \leq 0.05$; ${ }^{d} p \leq 0.01$

372 Table 4: Differences in social and other 'luxury' behavior identified between pair-matched cows with

373 (SCM) or without (CTRL) sub-clinical mastitis during 60 mins following morning milking 
(1hPostM1): parametric data (P) analysed using paired t-test, non-parametric data (NP) analysed using Wilcoxon SR test.

\begin{tabular}{|l|c|c|c|c|c|c|c|c|}
\hline \multirow{2}{*}{ Measure } & \multirow{2}{*}{ Test } & \multicolumn{2}{|c|}{ CTRL } & \multicolumn{2}{c|}{ SCM } & \multicolumn{3}{c|}{ Effect Size Statistics } \\
\cline { 3 - 9 } & & Mean & SD & Mean & SD & Hedges' g & $\mathbf{9 5 \% C I}$ & $\begin{array}{c}\text { Size of } \\
\text { effect }^{2}\end{array}$ \\
\hline SocR, (n) & NP & $12.1^{\mathrm{a}}$ & 7.43 & $6.6^{\mathrm{c}}$ & 3.92 & -0.91 & $0.17-1.51$ & Large, sig \\
\hline FocSocG, (n) & $\mathrm{P}$ & $10.7^{\mathrm{a}}$ & 9.0 & $5.8^{\mathrm{b}}$ & 4.7 & -0.70 & $-0.05-1.45$ & Med, ns \\
\hline FocSocR, (n) & $\mathrm{P}$ & $4.7^{\mathrm{a}}$ & 2.6 & $2.5^{\mathrm{c}}$ & 1.9 & -0.99 & $0.04-1.93$ & Large, sig \\
\hline BPR, (n) & $\mathrm{NP}$ & $3.3^{\mathrm{a}}$ & 2.5 & $1.2^{\mathrm{c}}$ & 1.5 & -0.98 & $0.29-1.67$ & Large, sig \\
\hline HBG, (n) & $\mathrm{NP}$ & $1.7^{\mathrm{a}}$ & 2.4 & $0.5^{\mathrm{b}}$ & 0.6 & -0.65 & $0.10-1.09$ & Med, sig \\
\hline HBR, (n) & $\mathrm{NP}$ & $1.1^{\mathrm{a}}$ & 1.4 & $0.3^{\mathrm{b}}$ & 0.6 & -0.73 & $0.10-1.40$ & Med, sig \\
\hline HPG, (n) & $\mathrm{NP}$ & $0.9^{\mathrm{a}}$ & 1.3 & $0.1^{\mathrm{c}}$ & 0.3 & -0.84 & $0.24-1.38$ & Large, sig \\
\hline ExpSoc, (s) & $\mathrm{NP}$ & $21.2^{\mathrm{a}}$ & 28.8 & $6.5^{\mathrm{c}}$ & 7.6 & -0.68 & $0.06-1.07$ & Med, sig \\
\hline AlloR, (s) & $\mathrm{NP}$ & $37.6^{\mathrm{a}}$ & 66.1 & $1.9^{\mathrm{c}}$ & 3.2 & -0.74 & $0.44-1.11$ & Med, sig \\
\hline Self-groom (s) & $\mathrm{P}$ & $29.2^{\mathrm{a}}$ & 25.6 & $47.4^{\mathrm{b}}$ & 44.2 & 0.52 & $0.01-1.03$ & Medium, sig \\
\hline ExpEnv, (s) & $\mathrm{P}$ & $71.8^{\mathrm{a}}$ & 44.2 & $39.3^{\mathrm{c}}$ & 27.8 & -0.91 & $0.11-1.71$ & Large, sig \\
\hline
\end{tabular}

${ }^{\mathrm{a}-\mathrm{c}}$ Mean values in the same row with different superscripts differ as follows: ${ }^{b} p<0.10$ (trend); ${ }^{\mathrm{c}} \mathrm{p} \leq 0.05$.

${ }^{1}$ SocR $=$ all social behavior received; FocSocG $=$ agonistic behavior given; FocSocR = agonistic behavior received; $\mathrm{BPR}=$ body push receive; $\mathrm{HBG}=$ head butt give; $\mathrm{HBR}=$ head butt receive; HPG = head push give; ExpSoc $=$ explore social; AlloR $=$ allogroom receive; Self-groom $=$ perform self-grooming behavior; ExpEnv $=$ explore environment.

${ }^{2}$ The size of the effect (based upon estimated g-values): $\leq 0.39=$ small, $0.40-0.79=$ medium, $\geq 0.80=$ large; significance attributed only when $95 \% \mathrm{CI}>0$ (i.e. do not contain ' 0 ')

(c) Social reactivity, social exploration, social proximity, and feed barrier preference

Over the $24 \mathrm{~h}$ period, cows with subclinical mastitis were also significantly less 'socially reactive' than healthy cows (Table 3), i.e. were less likely to move away/be displaced following the receipt of agonistic interactions (see Table 1). They performed significantly less social exploration than healthy controls ('ExpSoc') during both the $24 \mathrm{~h}$ period, and the 60 minutes following morning milking (Tables 3 and 4), and spent a significantly greater proportion of their time at the open section of the feed barrier over the $24 \mathrm{~h}$ period (Table 3 ). Cows with subclinical mastitis spent a significantly greater proportion of their time at the feed barrier flanked by two neighbours over the $24 \mathrm{~h}$ period than healthy controls (Table 3 ). On the basis of RDV confidence intervals, non-significant measures of social proximity were classified as biologically unimportant within the context of this study. This included proportion of time at the feed barrier without neighbors (' $\% \mathbf{F B} \_0 N N$ ': RDV $=8.28 \%$, 
398

399

400

401

402

403

404

405

406

407

408

409

410

411

412

413

414

415

416

417

418

419

420

421

422

423

424

425

426

427

428

429

430

431

two neighbors ('\%C_oNN': RDV $=7.75 \%, 95 \% \mathrm{CI}_{\mathrm{RDV}}=-0.06$ to $2.58 \%$; ' $\%$ \%C_2NN': RDV: $4.38 \%, 95 \% \mathrm{CI}_{\mathrm{RDV}}=-2.69$ to $\left.1.93 \%\right)$.

\section{(d) Allogrooming}

Over the $24 \mathrm{~h}$ period, SCM cows performed allogrooming significantly less than healthy controls (Table 3). Although receipt of allogrooming was not significantly different during $24 \mathrm{~h}$, and this measure was classified as biologically unimportant on the basis of RDV confidence intervals ('AlloR': $\mathrm{RDV}=11.02 \mathrm{~s}, 95 \% \mathrm{CI}_{\mathrm{RDV}}=-1.14$ to $1.67 \mathrm{~s}$ ), during 1hPostM1 the SCM cows were allogroomed less than the healthy controls (Table 4).

\subsubsection{Luxury behavior: Non-social}

(a) Self-grooming and brush use

In the 60 minutes following morning milking there was a tendency for SCM cows to perform more self-grooming than healthy controls (Table 4). For the $24 \mathrm{~h}$ period, differences in selfgrooming and brush use were not significant but classified as biologically inconclusive on the basis of confidence intervals for effect size differences ('Brush': $\mathrm{RDV}=8.70 \mathrm{~s}, 95 \% \mathrm{CI}_{\mathrm{RDV}}=$ -2.74 to $16.45 \mathrm{~s}$; 'Self-Groom': $\mathrm{RDV}=4.23 \mathrm{~s}, 95 \% \mathrm{CI}_{\mathrm{RDV}}=-2.81$ to $5.42 \mathrm{~s}$ ).

\section{(b) Environmental exploration}

In the 60 minutes following morning milking SCM cows explored the environment significantly less than healthy controls (Table 4). This difference was not evident over the $24 \mathrm{~h}$ period (Table 3), instead being classified as biologically unimportant in the context of this study, as based upon RDV confidence intervals ('ExpEnv': RDV $=11.08 \mathrm{~s}, 95 \% \mathrm{CI}_{\mathrm{RDV}}=$ -1.30 to $4.66 \mathrm{~s})$.

\subsubsection{Core maintenance behavior}

\section{(a) Feeding, drinking, and lying}

For both the $24 \mathrm{~h}$ observations and the 60 minutes following morning milking, no significant differences emerged in time spent feeding ('Feed'), drinking ('Drink'), or lying ('Lie') (Tables 5 and 6), nor was subclinical mastitis considered to have biologically important (or even inconclusive) effects on these behaviors over $24 \mathrm{~h}$ ('Feed': $\mathrm{RDV}=82.39 \mathrm{~s}, 95 \% \mathrm{CI}_{\mathrm{RDV}}=$ -0.03 to $0.03 \mathrm{~s}$; 'Drink': $\mathrm{RDV}=6.86 \mathrm{~s}, 95 \% \mathrm{CI}_{\mathrm{RDV}}=-1.33$ to $3.99 \mathrm{~s}$; 'Lie': RDV $=200.19 \mathrm{~s}$, $95 \% \mathrm{CI}_{\mathrm{RDV}}=-23.54$ to $79.67 \mathrm{~s}$ ). However, over the $24 \mathrm{~h}$ period $\mathrm{SCM}$ cows spent significantly more time lying with their head on their flank than healthy controls ('HOF', Table 5). 
(b) Physical activity

434 During both the $24 \mathrm{~h}$ period, and 60 minutes following morning milking, the SCM cows were significantly less active than healthy controls; SCM cows performed fewer behavioral transitions ('Trans') and moved over a smaller distance ('Dist') (Tables 5 and 6).

Table 5: Measures of 'core' maintenance behavior recorded from pair-matched cows with (SCM) or without (CTRL) sub-clinical mastitis over 24h: analysed using paired t-tests. All units transformed to 'per hour visible'.

442

443

444

445

446

447

448

449

450

451

452

453

454

455

456

457

\begin{tabular}{|l|c|c|c|c|c|c|c|}
\hline \multirow{2}{*}{$\begin{array}{c}\text { Measure } \\
\text { (unit) }\end{array}$} & \multicolumn{2}{|c|}{ CTRL } & \multicolumn{2}{c|}{ SCM } & \multicolumn{3}{c|}{ Effect size statistics } \\
\cline { 2 - 8 } & Mean & SD & Mean & SD & $\begin{array}{c}\text { Hedges' } \\
\text { g }\end{array}$ & 95\%CI & $\begin{array}{c}\text { Size of } \\
\text { effect }^{2}\end{array}$ \\
\hline Trans $^{\ddagger},(\mathrm{n})$ & $55.6^{\mathrm{a}}$ & 8.2 & $48.6^{\mathrm{c}}$ & 7.8 & 0.91 & $0.08-1.74$ & Large, sig \\
\hline Dist $^{\ddagger}$, (unit) & $8.2^{\mathrm{a}}$ & 1.9 & $6.8^{\mathrm{c}}$ & 1.5 & 0.88 & $0.11-1.65$ & Large, sig \\
\hline Feed $^{\dagger}$, (s) & $823.9^{\mathrm{a}}$ & 167.5 & $823.8^{\mathrm{a}}$ & 281.2 & 0.00 & $-0.46-0.46$ & Small, ns \\
\hline Drink $^{\dagger},(\mathrm{s})$ & $34.3^{\mathrm{a}}$ & 12.2 & $30.2^{\mathrm{a}}$ & 14.6 & 0.32 & $-0.32-0.96$ & Small, ns \\
\hline Lie $^{\dagger}$, (s) & $2001.9^{\mathrm{a}}$ & 211.6 & $2092.5^{\mathrm{a}}$ & 367.6 & -0.31 & $-0.26-0.88$ & Small, ns \\
\hline HOF $^{\ddagger},(\mathrm{s})$ & $143.5^{\mathrm{a}}$ & 49.7 & $178.7^{\mathrm{c}}$ & 74.8 & -0.57 & $0.06-1.09$ & Med, sig \\
\hline
\end{tabular}

${ }^{\mathrm{a}-\mathrm{c}}$ Mean values in the same row with different superscripts differ as follows: ${ }^{\mathrm{b}} \mathrm{p}<0.10$ (trend); ${ }^{\mathrm{c}} \mathrm{p} \leq 0.05$.

${ }^{1}$ Trans = behavioral transitions; Dist $=$ distance moved; Lie = lying in cubicle; HOF $=$ lie with head on flank.

${ }^{2}$ The size of the effect (based upon estimated g-values): $\leq 0.39=$ small, $0.40-0.79=$ medium, $\geq 0.80=$ large; significance attributed only when $95 \% \mathrm{CI}>0$

Number of data points used in statistical analysis: ${ }^{\dagger} \mathrm{n}=34 ;{ }^{\ddagger} \mathrm{n}=32$

Table 6: Differences in 'core' maintenance behavior identified between cows with (SCM) or without (CTRL) sub-clinical mastitis during 60 mins following morning milking (1hPostM1): parametric data (P) analysed using paired t-test, non-parametric data (NP) analysed using Wilcoxon SR test.

\begin{tabular}{|l|c|c|c|c|c|c|c|c|}
\hline \multirow{2}{*}{$\begin{array}{l}\text { Measure } \\
\text { (unit) }\end{array}$} & \multirow{2}{*}{ Unit } & \multicolumn{2}{|c|}{ Control } & \multicolumn{2}{c|}{ SCM } & \multicolumn{3}{c|}{ Effect size statistics } \\
\cline { 3 - 8 } & & Mean & $S D$ & Mean & $S D$ & $\begin{array}{c}\text { Hedge's g } \\
\text { (bootstrap) }\end{array}$ & 95\%CI & $\begin{array}{c}\text { Size of } \\
\text { effect }^{2}\end{array}$ \\
\hline Trans, (n) & NP & $99.1^{\mathrm{a}}$ & 34.8 & $71.7^{\mathrm{c}}$ & 24.6 & -0.89 & $0.21-1.62$ & Large, sig \\
\hline Dist, (unit) & $\mathrm{P}$ & $15.9^{\mathrm{a}}$ & 6.6 & $12.5^{\mathrm{b}}$ & 4.0 & -0.63 & $-0.08-1.34$ & Med, ns \\
\hline
\end{tabular}

${ }^{\mathrm{a}-\mathrm{c}}$ Mean values in the same row with different superscripts differ as follows: ${ }^{\mathrm{b}} \mathrm{p}<0.10$ (trend); ${ }^{\mathrm{c}} \mathrm{p} \leq 0.05$.

${ }^{1}$ Trans $=$ behavioral transitions; Dist $=$ distance moved.

${ }^{2}$ The size of the effect (based upon estimated g-values): $\leq 0.39=$ small, $0.40-0.79=$ medium, $\geq 0.80=$ large; significance attributed only when $95 \% \mathrm{CI}>0$

\subsection{Correlations Between Physiology and Behavior}




\subsubsection{Assay validation}

459 Parallelism $\left(\mathrm{F}_{1,9}=3.46, \mathrm{p}>0.05\right)$ was confirmed between serial dilutions of saliva (range: $1: 4$ 460 to $1: 64$ ) and SAA standards (range: $0,9.38,18.75,37.5,75,150,300 \mathrm{ng} / \mathrm{ml}$ ), indicating that 461 the ELISA kit was suitable for use with bovine saliva. Recovery of $300 \mathrm{ng} / \mathrm{ml} \mathrm{SAA}$ from a 462 spiked saliva sample was $93.76 \pm 4.63 \%(\mathrm{n}=10)$. The intra-assay CV was $3.09 \%(250.87 \pm$ $4637.75 \mathrm{ng} / \mathrm{ml}, \mathrm{n}=10)$ for $\mathrm{QC}_{\text {low }}$ and $4.68 \%(1360.33 \pm 63.70 \mathrm{ng} / \mathrm{ml}, \mathrm{n}=10)$ for $\mathrm{QC}_{\text {high. }}$ The 464 inter-assay CV was 2.77\% $(246.06 \pm 6.81 \mathrm{ng} / \mathrm{ml}, \mathrm{n}=2)$ for $\mathrm{QC}_{\text {low }}$ and 3.89\% $(1323.96 \pm$ $46551.43 \mathrm{ng} / \mathrm{ml}, \mathrm{n}=2$ ) for $\mathrm{QC}_{\text {high }}$.

466

\subsubsection{SAA and SCC}

468 The average SCC per group was: CTRL $=48.29 \pm 28.33(\mathrm{x} 1000$ cells $/ \mathrm{ml}) ; \mathrm{SCM}=351.12 \pm$ 469176.73 (x1000 cells $/ \mathrm{ml})$. A trend was found towards a significantly higher concentration of 470 salivary SAA in the SCM cows $(\mathrm{CTRL}=343.42 \pm 269.60 \mathrm{ng} / \mathrm{ml}, \mathrm{SCM}=519.59 \pm 315.43$ $\left.471 \mathrm{ng} / \mathrm{ml} ; \mathrm{t}_{1,12}=1.93, \mathrm{p}=0.076\right)$. A weak positive relationship was evident between SCC and 472 salivary SAA levels $\left(\mathrm{F}_{1,29}=8.81, \mathrm{p}=0.006\right.$, Figure 2$)$.

473

INSERT FIGURE 2 NEAR HERE

475

\subsubsection{SAA, SCC and behavior}

477 All behavioral measures significantly correlated with SAA and/or SCC are presented in Table

478 8. The majority of these relationships are weak as indicated by $\mathrm{R}^{2}$ values.

$480 \quad$ (a) $\quad \underline{\text { Total social interactions and agonistic interactions }}$

481 Significant positive relationships were found between salivary SAA and total social interactions received ('SocR') and total agonistic interactions received ('FocSocR'), indicating that cows with higher levels of salivary SAA cows received more social interactions in total, and also more agonistic interactions. Significant negative correlations were found between salivary SAA and total social interactions given ('SocG') and head swipes given ('HSG'), with a trend towards significance for total agonistic interactions given

487 ('FocSocG'). This indicates that cows with higher levels of salivary SAA cows initiated 488 fewer social interactions in total, including fewer agonistic interactions.

489 Table 8: Significant correlations $(p<0.05$; plus trends towards a correlation at $p<0.10)$ between 490 behavioral measures (24h data) and two markers of inflammation and mastitis infection, one from 491 saliva (serum amyloid-A, SAA), and one from milk (somatic cell counts, SCC); given are curve 
492

493

494

estimation regression statistics (ANOVA, coefficient of determination) and the equation for the relationship (based upon the line of best fit).

\begin{tabular}{|c|c|}
\hline $\begin{array}{l}\text { Behavioral measure }^{1} \\
\text { (unit) }\end{array}$ & Correlation with salivary SAA and SCC \\
\hline SocG (n) & SAA: $\mathrm{F}_{(1,29)}=6.42, \mathrm{p}=0.017 ; \mathrm{R}^{2}=0.181 ; \mathrm{y}=6.0091 \mathrm{e}^{-7 \mathrm{E}-04 \mathrm{x}}$ \\
\hline SocR (n) & $\begin{array}{l}\text { SAA: } F_{(2,28)}=7.40, p=0.003 ; R^{2}=0.346 ; y=9 E-06 x^{2}-0.0061 x+5.057 \\
\text { SCC: } F_{(2,31)}=4.90, p=0.014 ; R^{2}=0.240 ; y=2 E-05 x^{2}-0.0081 x+5.259\end{array}$ \\
\hline FocSocG (n) & SAA: $\mathrm{F}_{(1,29)}=3.07, \mathrm{p}=0.090 ; \mathrm{R}^{2}=0.096 ; \mathrm{y}=4.2715 \mathrm{e}^{-6 \mathrm{E}-04 \mathrm{x}}$ \\
\hline FocSocR (n) & SAA: $\mathrm{F}_{(2,28)}=4.23, \mathrm{p}=0.025 ; \mathrm{R}^{2}=0.232 ; \mathrm{y}=6 \mathrm{E}-06 \mathrm{x}^{2}-0.004 \mathrm{x}+3.817$ \\
\hline $\mathbf{C R}(\mathrm{n})$ & SAA: $\mathrm{F}_{(1,29)}=3.93, \mathrm{p}=0.057 ; \mathrm{R}^{2}=0.119 ; \mathrm{y}=0.0003 \mathrm{x}+0.165$ \\
\hline HPR (n) & SCC: $\mathrm{F}_{(1,31)}=5.87, \mathrm{p}=0.021 ; \mathrm{R}^{2}=0.159 ; \mathrm{y}=-1.990 \mathrm{x}+5.520$ \\
\hline HSG (n) & SAA: $\mathrm{F}_{(1,29)}=6.89, \mathrm{p}=0.014 ; \mathrm{R}^{2}=0.192 ; \mathrm{y}=2.3287 \mathrm{e}^{-9 \mathrm{E}-04 \mathrm{x}}$ \\
\hline HSR (n) & SCC: $F_{(2,30)}=3.14, p=0.058 ; R^{2}=0.173 ; y=6 E-06 x^{2}-0.0018 x+1.621$ \\
\hline \%FocSocR+D (\%) & $\begin{array}{l}\text { SAA: } F_{(1,29)}=4.63, p=0.040 ; R^{2}=0.138 ; y=-0.0155 x+52.078 \\
\text { SCC: } F_{(1,32)}=10.46, p=0.003 ; R^{2}=0.246 ; y=49.136 e^{-7 E-04 x}\end{array}$ \\
\hline$\% H B R+D(\%)$ & $\begin{array}{l}\text { SAA: } F_{(1,29)}=5.64, p=0.024 ; R^{2}=0.163 ; y=72.616 e^{-5 E-04 x} \\
\text { SCC: } F_{(1,31)}=8.81, p=0.006 ; R^{2}=0.221 ; y=-7.36 \ln (x)+95.594\end{array}$ \\
\hline$\% H P R+D(\%)$ & SAA: $\mathrm{F}_{(2,22)}=4.14, \mathrm{p}=0.030 ; \mathrm{R}^{2}=0.227 ; \mathrm{y}=-0.0002 \mathrm{x}^{2}+0.1952 \mathrm{x}-0.092$ \\
\hline$\% H S R+D(\%)$ & SAA: $\mathrm{F}_{(1,29)}=3.291, \mathrm{p}=0.080 ; \mathrm{R}^{2}=0.102 ; \mathrm{y}=-0.015 \mathrm{x}+34.655$ \\
\hline ExpSoc (s) & $\begin{array}{l}\text { SAA: } \mathrm{F}_{(1,29)}=4.34, \mathrm{p}=0.046 ; \mathrm{R}^{2}=0.130 ; \mathrm{y}=0.0167 \mathrm{x}+8.517 \\
\text { SCC: } \mathrm{F}_{(1,31)}=5.58, \mathrm{p}=0.025 ; \mathrm{R}^{2}=0.153 ; \mathrm{y}=15.532 \mathrm{e}^{-0.002 \mathrm{x}}\end{array}$ \\
\hline \%FB_Open (\%) & $\begin{array}{l}\text { SAA: } \mathrm{F}_{(1,28)}=4.08, \mathrm{p}=0.053 ; \mathrm{R}^{2}=0.127 ; \mathrm{y}=3.244 \ln (\mathrm{x})+67.752 \\
\text { SCC: } \mathrm{F}_{(1,32)}=5.98, \mathrm{p}=0.020 ; \mathrm{R}^{2}=0.158 ; \mathrm{y}=81.19 \mathrm{e}^{0.0003 \mathrm{x}}\end{array}$ \\
\hline ExpEnv (s) & SAA: $\mathrm{F}_{(1,29)}=5.96, \mathrm{p}=0.021 ; \mathrm{R}^{2}=0.170 ; \mathrm{y}=8.690 \ln (\mathrm{x})+7.484$ \\
\hline Trans (n) & SCC: $F_{(2,30)}=5.50, p=0.009 ; R^{2}=0.269 ; y=0.002 x^{2}-0.0934 x+59.272$ \\
\hline Dist (unit) & SCC $: F_{(2,30)}=4.11, p=0.026 ; R^{2}=0.215 ; y=4 E-05 x^{2}-0.021 x+9.146$ \\
\hline Feed $(\mathrm{s})$ & SAA: $\mathrm{F}_{(1,29)}=14.63, \mathrm{p}=0.001 ; \mathrm{R}^{2}=0.335 ; \mathrm{y}=1023.5 \mathrm{e}^{-6 \mathrm{E}-04 \mathrm{x}}$ \\
\hline Drink (s) & SAA: $\mathrm{F}_{(1,29)}=22.18, \mathrm{p}<0.001 ; \mathrm{R}^{2}=0.433 ; \mathrm{y}=-8.379 \ln (\mathrm{x})+79.816$ \\
\hline Lie (s) & $\begin{array}{l}\text { SAA: } F_{(1,29)}=9.10, p=0.005 ; R^{2}=0.239 ; y=0.5032 x+1848.3 \\
\text { SCC: } F_{(2,31)}=2.637, p=0.088 ; R^{2}=0.145 ; y=-0.0019 x^{2}+1.1955 x+1956.8\end{array}$ \\
\hline
\end{tabular}

${ }^{1} \mathrm{SocG}=$ all social behavior given; SocR $=$ all social behavior received; FocSocG $=$ agonistic behavior given; FocSocR = agonistic behavior received; $\mathrm{CR}=$ non-contact challenge receive; $\mathrm{HPR}=$ head push receive; $\mathrm{HSG}=$ head swipe give; HSR = head swipe receivd; \%FocSocR $+\mathrm{D}=$ percentage of occasions when receipt of agonism lead to displacement; $\% \mathrm{HBR}+\mathrm{D}=$ percentage of occasions when head butt receipt lead to displacement; $\% \mathrm{HPR}+\mathrm{D}=$ percentage of occasions when head push receipt lead to displacement; $\% \mathrm{HSR}+\mathrm{D}=$ percentage of occasions when head swipe receipt lead to displacement; ExpSoc $=$ explore social; \%FB_Open = percentage of time at feed barrier: open rail; ExpEnv $=$ explore environment; Trans = behavioral transitions; Dist $=$ distance moved. 
504 The significant relationship between SCC and total social interactions received ('SocR') was 505 best modelled using a quadratic function. This described a negative association at low SCC 506 levels and a positive association at higher levels, such that as cell counts rose in cows with 507 low SCC (i.e. healthy CTRL cows) the tendency to receive social interactions would 508 decrease, but in those cows with higher SCC (i.e. SCM cows) as cell counts rose further the 509 tendency to receive social interactions increased. The significant positive correlation between 510 SCC and the receipt of head swipes ('HSR') indicates that cows with higher SCC received 511 more agonistic head swipes.

512

\section{$513 \quad$ (b) Social reactivity}

514 Significant negative relationships were found between measures of social reactivity, (i.e. the

515 likelihood that a cow would be displaced/move away after receiving an agonistic interaction), 516 and both inflammatory markers. This is indicative that cows with higher levels of salivary 517 SAA and/or SCC demonstrate a reduced tendency to be displaced following the receipt of 518 social stimuli, including total agonistic interactions ( $\%$ FocSocR+D '), and specifically to 519 head butts ('\%HBR+D') (SAA and SCC), and head swipes received ('\%HSR+D') (SAA).

521 (c) Social and environmental exploration, and feed barrier preference

522 Salivary SAA was significantly positively associated with both social and environmental 523 exploration, such that cows with higher SAA levels performed more exploratory behavior.

524 SCC levels, however, were significantly negatively associated with social exploration, such that cows with higher cell counts explored other cows less. Salivary SAA and SCC were significantly positively correlated with proportion of time spent at the open-rail feed barrier ('\%FB_Open'), indicating that with rising levels of either inflammation measure, cows became more likely to feed at the open-rail feed barrier rather than the self-locking barrier.

(d) Physical activity, feeding, drinking, and lying

531 The significant relationships between SCC and both the number of behavioral transitions and distance covered were best modelled using quadratic functions. These describe an initial decline in both measures of physical activity as SCC increased to approximately 300 (x1000 cells/ml), followed by an increase in the measures as SCC levels continued to rise (Figure 3). significantly positively correlated with time spent lying; this indicates that, within our study 
538

539

540

541

542

543

544

545

546

547

548

549

550

551

552

553

554

555

556

557

558

559

560

561

562

563

564

565

566

567

568

569

cohort, that as SAA levels increased the cows spent less time feeding and drinking, and more time lying.

\section{DISCUSSION}

The purpose of this study was to identify potential changes in salivary serum amyloid-A (SAA) and behavior in cows with spontaneously occurring subclinical mastitis as compared to pair-matched, healthy controls. Salivary SAA was found to be higher in individuals with subclinical mastitis, suggesting that it may have potential as a marker of low-level systemic inflammation in dairy cows. Higher salivary SAA levels were also correlated with several predicted behavioral changes. Furthermore, cows with subclinical mastitis displayed a reduction in the performance and receipt of various social behaviours (here categorised as 'luxury' behaviors), lower 'social reactivity' (i.e. they were less likely to be displaced following the receipt of agonism), a reduction in physical activity, but no difference in feeding, drinking or lying duration ('core maintenance' behaviors).

\subsection{Changes in salivary SAA}

We report here, for the first time, a positive (although weak) correlation between SCC and salivary SAA. Positive associations between SCC and non-salivary SAA have previously been reported from cows with clinical and sub-clinical mastitis (serum: de Boyer Des Roches et al., 2017; milk: O’Mahony et al., 2006; Akerstedt et al., 2007; Pyörälä et al., 2011). SAA in saliva thus offers potential as a non-invasive means of detecting subclinical mastitis, but further studies will be required to substantiate our preliminary findings. Different bacterial strains, for example, can cause mastitis of different duration and degree (Verbeke et al., 2014), and it is possible that the concentration of SAA in saliva will vary accordingly. A case in point is Pyörälä et al.’s (2011) finding of significant differences in SAA (milk) collected from cows with spontaneous mastitis caused by different pathogens: low SAA was associated with $A$. pyogenes, while high concentrations were associated with $E$. coli. Consequently, further investigations will need to establish how, and when, salivary SAA levels change over the time course of an infection, with different pathogens, to substantiate its diagnostic potential as an inflammatory marker for mastitis and other diseases.

\subsection{Changes in Luxury Behaviors and correlations with salivary SAA}


570 'Luxury' behaviors, as used here, are those deemed non-essential for survival within the 571 short-term and, as such, we predicted that during a subclinical infection they would be downregulated. Within this category we included both social and non-social behaviors.

\subsubsection{Social interactions}

575 Over the $24 \mathrm{~h}$ period, total social behavior, given or received, remained unaltered between the 576 two groups. Subclinically mastitic cows did, however, receive fewer non-contact challenges

577 and more head swipes. Head swipes are most commonly observed at the feed barrier as a

578 means of displacing competitors, and our result is in agreement with previous findings. Sick 579 cows are typically reported to be displaced more frequently than healthy cows when feeding, 580 as would result from head swipes or other agonistic behavior (Schirmann et al., 2016; Lomb et al., 2018; Neave et al., 2018;).

582

583 In the 60 minutes following morning milking, that is during the period of peak feeding 584 activity in the study herd, other differences emerged; cows with subclinical mastitis received 585 fewer social interactions in total, and specifically fewer head butts and body pushes, than the

586 healthy controls. No corresponding reduction in the receipt of agonistic interactions involving 587 physical contact were observed over the $24 \mathrm{~h}$ period. It is therefore possible that the subclinically mastitic cows were avoiding peak feeding when aggression and competition for feed are highest, as do low-ranking cows (Val-Laillet et al. 2008).

Interestingly, we also found a positive correlation between salivary SAA and total social interactions, and agonistic interactions, received, indicating that those individuals with higher levels of systemic inflammation received more agonistic interactions. However, we cannot be sure whether SAA upregulation occurred in the cows with subclinical mastitis due to infection or following exposure to social stress. Upregulation of C-Reactive Protein (another acute phase protein known to increase during illness and stress) has, for example, been reported in zoo-housed gorillas following an aggressive encounter (Fuller \& Allard, 2018). In the current study, saliva samples were collected the day after behavioral video recordings. Therefore, an elevation in SAA could reflect agonistic encounters experienced during the previous day, independent of health status. Further study should address this potential confound. 
603 In line with the prediction that social behaviors should decrease with subclinical mastitis,

604 affected cows performed fewer head butts than healthy cows, and SAA was negatively (albeit

605 loosely) correlated with the delivery of total social and agonistic interactions, including head

606 swipes in particular. Our results thus provide additional behavioral detail in support of

607 existing literature which reports that cows with clinical (and subclinical) conditions often

608 perform fewer agonistic interactions and competitive displacements from the feed-bunk

609 (Galindo \& Broom, 2002; Huzzey et al., 2007; Patbandba et al., 2012; Sepúlveda-Varas et al.,

$6102014 ; 2016$ ), and from cubicles (Jensen \& Proudfoot, 2017), than healthy individuals.

611

612 The relative social ranks of study cows and their interaction partners were not determined in

613 the current study. It is possible, and an interesting focus for further study, that the relative

614 social rank of an individual could be influenced by the effects of disease due to a loss of

615 competitive vigour and motivation. High-ranking animals characteristically displace lower

616 rankers cows from the feed barrier (DeVries et al., 2004; Huzzey et al., 2006), and lower

617 rankers adjust their feeding patterns accordingly (DeVries et al., 2004).

618

\section{4.2.2. Social reactivity}

620 We found that over the $24 \mathrm{~h}$ period, cows with subclinical mastitis were less likely to move away/be displaced than matched healthy cows following the receipt of agonism, labelled here as less 'socially reactive'. Correspondingly, reactivity declined with increasing inflammation levels as demonstrated by a negative correlation between SAA levels and percentage displacement following the receipt of agonistic interactions overall, and specifically following the receipt of head butts and head swipes. Similarly, SCC was negatively correlated with the percentage displacement following receipt of agonistic interactions overall, and specifically of head butts, such that cows with higher SCC were less likely to more away after being head butted, and/or following receipt of all agonistic interactions, than cows with lower counts. These results appear, at first hand, to contradict previous findings of sick cows being less motivated to compete for access to feed based on displacement (e.g. Huzzey et al, 2006; 2007; Goldhawk et al., 2009); however, where a cow positions herself at the feeder may, in turn, be influenced by the social rank of others already feeding, as reported by Manson \& Appleby (1990). They found that cows of the most dissimilar rank (low/high) maintained a greater distance from each other than cows of similar rank. It is thus 
636 individuals of higher competitive vigour, and preferentially selected the company of other

637 sick or lower ranking cows. Subsequently, the receipt of moderate competitive aggression

638 from an individual, with similarly moderate motivation to compete, may have been tolerable

639 and thus not provoked displacement. This is beyond the reach of the current data as we did

640 not determine the relative social ranks of our focal cows and their interaction partners, but

641 warrants further investigation. As others before us, we can therefore not rule out that this and

642 our other findings on social behavioral differences reflect underlying differences in social

643 status that have led to differences in health status. We here provide a first report of a

644 reduction in social reactivity in cows with subclinical mastitis. By matching cows by parity

645 we sought some control over potentially confounding social rank biases. Further study is

646 needed to substantiate differences in social interactions as markers of subclinical mastitis and

647 other diseases.

648

649 4.2.3. Social avoidance and proximity

650 In the current study, cows with subclinical mastitis performed less social exploration than

651 healthy controls during both the $24 \mathrm{~h}$ period and the focussed $60 \mathrm{~min}$ following morning

652 milking, which indicates social avoidance or withdrawal. Furthermore, we observed a

653 negative correlation between this measure and SCC, such that cows with higher counts spent

654 less time exploring (sniffing) others. Sickness-driven social avoidance is well documented in

655 lab-animals and humans and is predicted via the action of pro-inflammatory cytokines on the

656 central nervous system (Kent et al., 1992; Bluthe et al., 1996; Dantzer \& Kelley, 2007;

657 Arakawa et al., 2010). Due to the limited opportunity for social avoidance to occur within

658 intensive systems it has been relatively understudied in farm animals. We also found an

659 unexpected, if weak, positive correlation between SAA and social exploration, which may, at

660 least partially, be explained by the presence of both pre-clinical and post-clinical individuals

661 amongst our subclinical group. De Boyer Des Roches et al. $(2017 ; 2018)$ report behavioral

662 changes including reduced environmental attentiveness during the pre-clinical phase of an

663 experimentally-induced mastitis model (i.e. prior to SCC and serum SAA upregulation), and

664 during the acute phase (coinciding with raised levels of SCC and SAA), but not during the

665 post-clinical remission phase (when high levels of SCC and SAA are evident). This suggests

666 that serum SAA may peak during post-clinical remission, rather than during the acute phase

667 of inflammation. Further study is therefore required to investigate when, and how, social 
668

669

670

671

672

673

674

675

676

677

678

679

680

681

682

683

684

685

686

687

688

689

690

691

692

693

694

695

696

697

698

699

700

exploration changes throughout the course of spontaneously-occurring mastitis, and other diseases, to confirm its usefulness in the detection of subclinical disease states.

We found that over the $24 \mathrm{~h}$ period, cows with subclinical mastitis spent a greater proportion of their time feeding at the open-rail section of the feed barrier than healthy controls. A weak positive correlation was also found between this measure and SAA, which suggests that this preference increases with systemic inflammation. Barrier design is known to influence the incidence of agonistic behavior relating to feed access. Self-locking yokes have vertical bars which separate the necks of adjacent cows, and these are better at reducing competitive interactions (usually measured by displacements) compared to open post-and-rail barriers (Endres et al., 2005; Huzzey et al., 2006). Our results therefore appear to contradict previous findings. However, other factors are likely to also contribute to the choice of feeding location. In the open section cows have better visibility and are more quickly able to withdraw from potential agonistic interactions. Our finding that subclinically mastitic cows spent a greater proportion of their time than the healthy cows at the feed barrier in close contact with two (flanking) herdmates may be at odds with the expectation of sickness-driven social avoidance. However, sick cows may also feel more vulnerable and therefore seek to feed in company. Future longitudinal studies could determine whether individuals change their feeding behavior in relation to health status, including whether they opt to feed amongst others of lower rank when their own health is compromised.

\subsubsection{Allogrooming}

In agreement with our predictions relating to the basis of behavioral priorities during infection, cows with subclinical mastitis performed less allogrooming than the healthy cows over the $24 \mathrm{~h}$ period, and during the 60 min following morning milking they themselves were also allogroomed less. Previous studies have reported similar findings in relation to social rank, with low-ranking cows performing and receiving less allogrooming than high rankers (Napolitano et al., 2009), and allogrooming decreasing more in low-ranking cows under conditions of increased competition (Val-Laillet et al., 2008). For health-related changes, Galindo \& Broom (2002) found lame cows to be allogroomed more than non-lame cows, which is contrary to our result in subclinically mastitic cows. Their finding was interpreted as a self-instigated coping strategy triggered by pain/discomfort associated with clinical lameness, which arguably applies less to subclinical mastitis. 
701 Since social grooming serves a variety of functions in cattle, including roles in hygiene,

702

703

704

705

706

707

708

709

710

711

712

713

714

715

716

717

718

719

720

721

722

723

724

725

726

727

728

729

730

731

732

733

734

pleasure, maintenance of social bonds and lowering social tension (Sato et al., 1991; 1993), it

is possible that prolonged suppression of allo-grooming, for example during a low-level

chronic inflammatory condition, could negatively affect cow welfare and health itself.

\subsubsection{Non-social behavior}

\section{(a) Self-grooming}

Against expectation, we did not find a difference in brush use between cows with and without subclinical mastitis, nor a correlation between this behavior and SAA. Although grooming itself is a comfort activity that healthy cows are highly motivated to perform (McConnachie et al., 2018), brush use has been shown to decrease during non-mastitic disease (Toaff Rosenstein et al., 2016; Mandel et al., 2017; Weigele et al., 2017). The brush in our study was located central to many resources including the feed barrier, a water trough and cubicles and was, therefore, readily accessible to all cows with minimal effort. In addition to our relatively small sample size, a trade-off between brush location and the sensitivity of brush use for detecting stress and morbidity (Mandel et al., 2013; 2017) may help to explain why no decline was detected in the current study.

We observed cows with subclinical mastitis to perform more self-grooming (including selflicking) than controls immediately following morning milking, presumably as a response to mild udder discomfort or as a substitute for allo-grooming (see section 4.2.4). However, this observation is at odds with existing literature that reports self-licking to remain unchanged (Siivonen et al., 2011), or even decrease (Fogsgaard et al., 2012), during clinical mastitis.

Based upon our results the potential for the use of self-grooming as a marker of subclinical mastitis remains inconclusive and requires further investigation.

\section{(b) Environmental exploration}

The weak positive correlation between SAA and environmental exploration described in the current study was unexpected since exploratory behavior was predicted to decline with increasing inflammation. As our study did not use an experimental model of induced mastitis we do not know whether our focal cows comprised a mixture of early-stage, pre-clinical mastitic cows (potentially associated with low SAA and sickness-driven reductions in environmental exploration), and individuals in post-clinical remission (potentially associated with high SAA and normal baseline values) (as discussed in Section 4.2.3). Ruminants 
735

736

737

738

739

740

741

742

743

744

745

746

747

748

749

750

751

752

753

754

755

756

757

758

759

760

761

762

763

764

765

766

767

768

generally display low baseline levels of exploratory behavior when maintained in intensive housing, since it is a largely unstimulating environment (De Rosa et al., 2009). Against expectation, we also did not find a reduction in environmental exploration in our subclinically mastitic cows over the 24 hours. They did, however, explore less than controls during the 60 min following morning milking. Environmental exploration, as defined in our study, included sniffing the feed as well as pen fittings and cubicle sand. Lower levels of exploration in cows with subclinical mastitis after morning milking may therefore also reflect avoidance of feeding at peak time.

\subsection{Core Maintenance Behavior}

\subsubsection{Activity}

Our finding that cows with subclinical mastitis made fewer behavioral transitions and moved over a shorter distance than healthy cows is in agreement with our predictions, and with other studies that describe reduced activity prior to the clinical diagnosis of mastitis (Kester et al., 2015; Stangaferro et al., 2016; Veissier et al., 2017; King et al., 2018). The quadratic relationships between SCC and both 'behavioral transitions' and 'distance moved' described in the current study are of interest because mastitic cows have also been reported to display increased activity (Siivonen et al., 2011; Medrano-Galarza et al., 2012), presumably due to udder discomfort and an associated reduction in lying time. Jadhav et al. (2018) argue that the threshold SCC value to delineate subclinical mastitis from normal should be 310 , rather than 200 (x1000 cells/ml), as conventionally judged (e.g. Madouasse et al., 2010). This higher value closely corresponds with the parabola vertex in both quadratic plots (Figure 3), of approx. $300(\mathrm{x} 1000 \mathrm{cells} / \mathrm{ml})$; i.e. the point at which activity once again begins to rise.

\subsubsection{Feeding/Drinking}

Changes in feeding behavior have long been used to diagnose the onset of illness (Weary et al., 2009). We found a negative correlation between SAA and feeding duration, as would be predicted with sickness. Although we observed a negative correlation between SAA and feeding duration, as would be hypothesised to occur with sickness, the average inflammatory response within our SCM group overall was not sufficiently pronounced to trigger obvious anorexia, as compared to CTRL cows. Sepúlveda-Varas et al. (2016) observed a decrease in feed intake (but not duration) prior to the diagnosis of clinical mastitis which may be attributed to underlying malaise. González et al. (2008) reported individual variability in feeding behavior relating to spontaneously occurring udder disorders. Some cows 
769 demonstrated a decrease in feeding duration with the onset of mastitis, while others showed no change. It is possible that aspects of feeding behavior, other than duration, may have been affected. Barn-housed cattle demonstrate highly synchronised feeding activity, with large peaks in both feeding and social competition coinciding with fresh food delivery, and smaller peaks following milking (DeVries \& von Keyserlingk, 2005; Dollinger \& Kaufmann, 2013). Mastitic cows, presumably to avoid adverse social interactions, have been shown to feed at less popular times such as early afternoon (Schirmann et al., 2016). While we found no direct effect on feeding duration, our findings on social interactions after morning milking, reported above, may be indicative of cows with subclinical mastitis avoiding peak feeding times.

Water and feed intake are positively related in cattle (Kume et al., 2010); however, drinking tends to be less affected by health than feeding (Hart, 1988). Water is more immediately vital for maintaining bodily functions (Kyriazakis and Tolkamp, 2011), and since drinking takes less time than food consumption it is less prone to being disrupted by social competition at the trough (Huzzey et al., 2007). Although a reduction in water consumption has been reported in cows with mastitis (Lukas et al., 2008; Siivonen et al., 2011), and we observed a moderate negative correlation between SAA and drinking duration, the level of systemic inflammation within our subclinically sick cows may have been too low, and/or our sample size too small, for a difference to emerge. Overall, the persistence of feeding and drinking levels in our subclnically mastitic cows fits the prediction that core maintenance behavior is conserved during early mastitic disease.

\subsubsection{Lying}

792

No difference in lying duration was found between our two groups. Lying is a highly prioritised behavior in cattle due to its importance in rumination (Jensen et al., 2004; 2005; Munksgaard et al., 2005) and dairy cows spend approximately $11 \mathrm{~h} /$ day recumbent (Ito et al., $2009 ; 2010)$. Increased lying duration, as a means of conserving energy and facilitating recovery, is a key adaptation for sickness, and a positive correlation between SAA and lying was found in our cows. Although extended lying duration has been frequently reported during non-mastitic clinic conditions (Toaff Rosenstein et al., 2016; Weigele et al., 2017; Barragan et al., 2018), lying may actually decrease during mastitis (Yeiser et al., 2012; Fogsgaard et al., 2012; 2015; Medrano-Galarza et al. 2012;), most likely due to udder pain (Cyples et al., 
803 We also observed that cows with subclinical mastitis lay with their heads held against their

804 flank more than the healthy controls. This posture is primarily associated with rapid eye movement (REM) sleep, but cows are also known to display non-rapid eye movement

806 (NREM) sleep and drowsing in this position (Ternman et al., 2013), and NREM (deep) sleep

807 often increases during infection (Bryant et al., 2004; Opp, 2005). Crucially, the action of pro-

808 inflammatory cytokines during the sickness response also predicts postural changes, such as

809 'curling up', that reduce surface area and associated loss of body heat. Lying with neck and

810 head bent back against and resting on the body achieves a reduction in surface area in cows,

811 and as such fits with predictions for adaptive behavioral changes during sickness. On the

812 basis of our study, lying duration itself appears less promising for the detection of sub-

813 clinical mastitis than our novel finding of a difference in lying posture.

814

\section{$815 \quad 4.4 \quad$ Limitations}

816 This study formed a preliminary investigation, to identify behaviors with potential for use as

817 a marker of sub-clinical mastitis, with the intention of informing a wider schedule of focused

818 research; longitudinal studies are now required to track changes in key behaviors with

819 deviations in the health status of individual cows. Due to an absence of pre-existing literature

820 (luxury behavior, in this context, has been understudied), a large number of measures were

821 recorded over 24h. Logistically this was time consuming and limited the amount of data

822 available per cow; priority was given to sample size. The analysis of a single $24 \mathrm{~h}$ period for

823 each cow provided a snapshot of one day, It is therefore not possible to conclude that

824 behaviors observed were typical of that cow at that level of SCC. Although we health-

825 checked study cows for inclusion in the study, as described in the Methods, to rule out

826 additional clinical pathologies we could not determine subclinical conditions other than

827 mastitis, nor could we establish whether cows classified as having subclinical mastitis were in

828 the pre-clinical phases of disease or in remission.

\section{$830 \quad 5 . \quad$ CONCLUSIONS}

831 By studying in detail the behavior of healthy cows and cows with spontaneously occurring subclinical mastitis, pair matched by reproductive parameters and parity, over two time periods (24h and 60 min following morning milking) we found that subclinical mastitis was

834 associated with reductions in activity, social exploration, the receipt of non-agonistic social

835 behavior, social reactivity (probability of displacement following receipt of agonism), and an

836 increase in the receipt of head swipes, compared to clinically healthy control cows. Many 
837 social interactions can be considered 'luxury' behaviors during sickness, and here we provide

838 preliminary findings that suggest that several social measures change at subclinical levels of

839 mastitis, as predicted, whereas 'core' maintenance activities (including feed, drink and lie)

840 did not, and luxury behaviors therefore offer greater potential for use in early disease

841 detection. The wider social context and, specifically the rank relationships between study

842 cows and their interaction partners, however, were beyond the scope of this investigation, and

843 further study is needed on the interactions between social rank, health status and behavior in 844 cows.

845

846 Cows with subclinical mastitis displayed a different feeding pattern. They spent a greater

847 proportion of their feeding time in direct contact with two neighbours, and a lower proportion

848 of time feeding at the self-locking feed barriers, than the healthy cows. Although a positive

849 relationship between SCC and salivary SAA was observed, and several correlations between

850 SAA and behavioral measures were identified in a direction consistent with sickness behavior

851 (including positive correlations with lying duration and the receipt of total agonism, and

852 negative correlations with feeding, drinking, the performance of total social and agonistic

853 behavior, and social reactivity), the majority of associations were relatively weak.

855 With this study, we have taken initial steps to identify physiological and detailed behavioral

856 changes associated with subclinical mastitis; a number of our findings are consistent with

857 predictions for low-level sickness responses. Social behavior is fundamentally dependent

858 upon the wider social environment and necessitates interactions between focal animals and

859 other individuals within the herd. We consider the preliminary identification of behavioral

860 differences in a small group of cows within a complex and dynamic social environment very

861 encouraging. We now recommend that observations be replicated in longitudinal studies,

862 tracking individuals, and larger data sets to substantiate and refine our findings.

\section{ACKNOWLEDGEMENTS}

865 This study was funded by The John Oldacre Foundation (grant code R12518-102). We would also like to thank the staff at Wyndhurst Farm for facilitating our research.

\section{COMPETING INTERESTS STATEMENT}

869 The authors have no competing interests to declare. 
883

884

885

886

887

888

889

890

891

892

893

894

895

896

897

898

899

900

901

902

903

904

905

906

907

908

909

910

911

912

913

914

915

916

917

918

919

\section{REFERENCES}

AHDB, 2015. The Agriculture and horticulture development board dairy mobility scoresheet https://projectblue.blob.core.windows.net/media/Default/Dairy/Publications/Dairy\%20Mobili ty\%20Scoresheet_200427_WEB.pdf - accessed 14/07/20

Åkerstedt, M., K. P. Waller, and A. Sternesjö, 2007. Haptoglobin and serum amyloid A in relation to the somatic cell count in quarter, cow composite and bulk tank milk samples. J. Dairy Res. 74:198-203. https://doi.org/10.1017/S0022029906002305

Andrew, W., J. Gao, S. M. Mullan, N. W. Campbell, A. Dowsey, and T. Burghardt, 2020. Visual identification of individual holstein friesian cattle via deep metric learning. Comput. Electron. Agric. https://arxiv.org/abs/2006.09205

Arakawa, H., K. Arakawa, and T. Deak, 2010. Sickness-related odor communication signals as determinants of social behavior in rat: a role for inflammatory processes. Horm. Behav. 57:30-341. https://doi.org/10.1016/j.yhbeh.2010.01.002

Barragan A. A., J. M. Piñeiro, G. M. Schuenemann, P. J. Rajala-Schultz, D. E. Sanders, J. Lakritz, and S. Bas, 2018. Assessment of daily activity patterns and biomarkers of pain, inflammation, and stress in lactating dairy cows diagnosed with clinical metritis. J. Dairy Sci. 101(9):8248-8258. https://doi.org/10.3168/jds.2018-14510

Bluthé, R. M., B. Michaud, K. W. Kelley, and R. Dantzer, 1996. Vagotomy attenuates behavioural effects of interleukin-1 injected peripherally but not centrally. Neuroreport 7(9):1485-1488. https://doi.org/10.1097/00001756-199606170-00008

Bryant, P. A., J. Trinder, and N. Curtis, 2004. Sick and tired: Does sleep have a vital role in the immune system? Nature Rev. Immunol. 4:457-467. https://doi.org/10.1038/nri1369

Cohen, J., 1988. Chapter 6: Differences between proportions. Pages 184-185 in Statistical Power Analysis for the Behavioral Sciences, 2nd edition. Hillsdale, Lawrence Erlbaum Publishers.

Cyples, J. A., C. E. Fitzpatrick, K. E. Leslie, T. J. DeVries, D. B. Haley, and N. Chapinal, 2012. Short communication: The effects of experimentally induced Escherichia coli clinical mastitis on lying behavior of dairy cows. J. Dairy Sci. 95(5):2571-2575.

https://doi.org/10.3168/jds.2011-5135.

Dantzer, R. 2004. Cytokine-induced sickness behaviour: A neuroimmune response to activation of innate immunity. Eur. J. Pharmacol. 500:399-411.

https://doi.org/10.1016/j.ejphar.2004.07.040

Dantzer, R. and K. W. Kelley, 2007. Twenty years of research on cytokine-induced sickness behaviour. Brain Behav. Immun. 21(2):153-160. https://doi.org/10.1016/j.bbi.2006.09.006

Dantzer, R., J. C. O'Connor, G. G. Freund, R. W. Johnson, and K. W. Kelley, 2008. From inflammation to sickness and depression: When the immune system subjugates the brain. Nature Rev. Neurosci. 9(1):46-56. https://doi.org/10.1016/j.bbi.2006.09.006 
920 Dawkins, M. S. 1990. From an animal's point of view: Motivation, fitness, and animal

921

922

923

924

925

926

927

928

929

930

931

932

933

934

935

936

937

938

939

940

941

942

943

944

945

946

947

948

949

950

951

952

953

954

955

956

957

958

959

960

961

962

963

964

965

966

967

968

969

welfare. Behav. Brain Sci. 13:1-9. https://doi.org/10.1017/S0140525X00077104

De Boyer des Roches, A. D. B., M. Faure, A. Lussert, V. Herry, P. Rainard, D. Durand, and G. Foucras, 2017. Behavioral and patho-physiological response as possible signs of pain in dairy cows during Escherichia coli mastitis: A pilot study. J. Dairy Sci. 100:8385-8397. https://doi.org/10.3168/jds.2017-12796

De Boyer des Roches, A. D. B., A. Lussert, M. Faure, V. Herry, P. Rainard, D. Durand, F. Wemelsfelder, and G. Foucras, 2018. Dairy cows under experimentally-induced Escherichia coli mastitis show negative emotional states assessed through Qualitative Behaviour Assessment. Appl. Anim. Behav. Sci. 206:1-11. https://doi.org/10.1016/j.applanim.2018.06.004

De Rosa, G., F. Grasso, A. Braghieri, A. Bilancione, A. Di Francia, and F. Napolitano, 2009. Behavior and milk production of buffalo cows as affected by housing system. J. Dairy Sci. 92(3):907-912. https://doi.org/10.3168/jds.2008-1157

DeVries, T. J. and M. A. G. von Keyserlingk, 2005. Time of feed delivery affects the feeding and lying patterns of dairy cows. J. Dairy Sci. 88: 625-631.

https://doi.org/10.3168/jds.S0022-0302(05)72726-0

DeVries, T. J., M. A. G. von Keyserlingk, and D. M. Weary, 2004. Effect of feeding space on the inter-cow distance, aggression, and feeding behavior of free-stall housed lactating dairy cows. J. Dairy Sci. 87:1432-1438. https://doi.org/10.3168/jds.S0022-0302(04)73293-2

Dollinger, J. and O. Kaufmann, 2013. Feeding behaviour in dairy cows with and without the influence of clinical diseases or subclinical disorders. Archiv. Tierzucht 56(14):149-159. https://doi.org/10.7482/0003-9438-56-014

Endres, M. I., T. J. DeVries, M. A. G. von Keyserlingk, and D. M. Weary, 2005. Effect of feed barrier design on the behavior of loose housed lactating dairy cows. J. Dairy Sci. 88:2377-2380. https://doi.org/10.3168/jds.S0022-0302(05)72915-5

Fogsgaard, K. K., C. M. Røntved, P. Sørensen, and M. S. Herskin, 2012. Sickness behavior in dairy cows during Escherichia coli mastitis. J. Dairy Sci. 95(2):630-638.

https://doi.org/10.3168/jds.2011-4350

Fogsgaard, K. K., T. W. Bennedsgaard, and M. S. Herskin, 2015. Behavioral changes in freestall-housed dairy cows with naturally occurring clinical mastitis. J. Dairy Sci. 98:17301738. https://doi.org/10.3168/jds.2014-8347

Fuller, G. and S. Allard, 2018. Preliminary data showing potential for salivary C-reactive protein as an indicator of welfare in western lowland gorillas (Gorilla gorilla gorilla). J. Vet. Behav. 28:58-62. https://doi.org/10.1016/j.jveb.2018.08.005

Galindo, F. and D. M. Broom, 2002. The effects of lameness on social and individual behavior of dairy cows. J. Appl. Anim. Welfare Sci. 5(3):193-201.

https://doi.org/10.1207/S15327604JAWS0503_03 
970 Gerlanc, D. and K. N. Kirby, 2012. bootES (Version 1.0). Retrieved from http://cran.r-

971

972

973

974

975

976

977

978

979

980

981

982

983

984

985

986

987

988

989

990

991

992

993

994

995

996

997

998

999

1000

1001

1002

1003

1004

1005

1006

1007

1008

1009

1010

1011

1012

1013

1014

1015

1016

1017

1018

project.org/web/packages/bootES/index.html

Gleerup, K. B., P. H. Andersen, L. Munksgaard, and B. Forkman, 2015. Pain evaluation in dairy cattle. Appl. Anim. Behav. Sci. 171:25-32.

https://doi.org/10.1016/j.applanim.2015.08.023

Goldhawk, C., N. Chapinal, D. M. Veira, D. M. Weary, and M. A. G. von Keyserlingk, 2009. Prepartum feeding behavior is an early indicator of subclinical ketosis. J. Dairy Sci. 92:49714977. https://doi.org/10.3168/jds.2009-2242

González, L. A., B. J. Tolkamp, M. P. Coffey, A. Ferret, and I. Kyriazakis, 2008. Changes in feeding behavior as possible indicators for the automatic monitoring of health disorders in dairy cows. J. Dairy Sci. 91:1017-1028. https://doi.org/10.3168/jds.2007-0530

Gu, J. Q., Z. H. Wang, R. H. Gao, H. R. Wu, 2017. Cow behavior recognition based on image analysis and activities. Int. J. Agric. Biol. Eng. 10(3):165-174. https://doi.org/10.165174.10.3965/j.ijabe.20171003.3080

Hart, B. L., 1988. Biological basis of the behavior of sick animals. Neurosci. Biobehav. Rev. 12:123-137. https://doi.org/10.1016/S0149-7634(88)80004-6

Hedges, L.V., 1981. Distributional theory for Glass's estimator of effect size and related estimators. J. Educ. Stats 6:107-128. https://doi.org/10.3102\%2F10769986006002107

Hoonhout, L., I. Reimert, and C. I. Daigle, 2017. Relationship between allogrooming and disease in feedlot steers: Social interactions may provide information about individual animal health. J. Anim. Sci. 95(s4):2. https://doi.org/10.2527/asasann.2017.003

Huzzey, J. M., T. J. DeVries, P. Valois, and M. A. G. von Keyserlingk, 2006. Stocking density and feed barrier design affect the feeding and social behavior of dairy cattle. J. Dairy Sci. 89:126-133. https://doi.org/10.3168/jds.S0022-0302(06)72075-6

Huzzey, J. M., D. M. Veira, D. M. Weary, and M. A. G. von Keyserlingk, 2007. Prepartum behavior and dry matter intake identify dairy cows at risk for metritis. J. Dairy Sci. 90:32203233. https://doi.org/10.3168/jds.2006-807

ISO 13366-1, 1997. Part 1: Microscopic method (Reference method). In: Milk - Enumeration of Somatic Cells. International Organization for Standardisation, Geneva, Switzerland. https://webstore.ansi.org/Standards/ISO/ISO133661997

Ito, K., D. M. Weary, and M. A. G. von Keyserlingk, 2009. Lying behavior: Assessing within- and between-herd variation in free-stall-housed dairy cows. J. Dairy Sci. 92:44124420. https://doi.org/10.3168/jds.2009-2235

Ito, K., M. A. G. Von Keyserlingk, S. J. LeBlanc, and D. M. Weary, 2010. Lying behavior as an indicator of lameness in dairy cows. J. Dairy Sci. 93(8):3553-3560.

https://doi.org/10.3168/jds.2009-2951 
1019 Jadhav, P. V., D. N. Das, K. P. Suresh, and B. R. Shome, 2018. Threshold somatic cell count

1020 for delineation of subclinical mastitis cases. Vet World 11(6):789-793.

1021

1022

1023

1024

1025

1026

1027

1028

1029

1030

1031

1032

1033

1034

1035

1036

1037

1038

1039

1040

1041

1042

1043

1044

1045

1046

1047

1048

1049

1050

1051

1052

1053

1054

1055

1056

1057

1058

1059

1060

1061

1062

1063

1064

1065

1066

1067 https://doi.org/10.14202/vetworld.2018.789-793.

Jensen, M. B. and K. L. Proudfoot, 2017. Effect of group size and health status on behavior and feed intake of multiparous dairy cows in early lactation. J. Dairy Sci. 100:9759-9768. https://doi.org/10.3168/jds.2017-13035

Jensen, M. B., L. Munksgaard, L.J. Pedersen, J. Ladewig, and L. Matthews, 2004. Prior deprivation and reward duration affect the demand function for rest in dairy heifers. Appl. Anim. Behav. Sci. 88:1-11. https://doi.org/10.1016/j.applanim.2004.02.019

Jensen, M. B., L. J. Pederson, and L. Munksgaard, 2005. The effect of reward duration on demand functions for rest in dairy heifers and lying requirements as measured by demand functions. Appl. Anim. Behav. Sci. 90:207-217.

https://doi.org/10.1016/j.applanim.2004.08.006

Kent, S., R. M. Bluthé, K. W. Kelley, and R. Dantzer, 1992. Sickness behavior as a new target for drug development. Trends in Pharmacol. Sci. 13:24-28.

https://doi.org/10.1016/0165-6147(92)90012-U

Kester, H. J., D. E. Sorter, and J. S. Hogan, 2015. Activity and milk compositional changes following experimentally induced Streptococcus uberis bovine mastitis. J. Dairy Sci. 98(2):999-1004. https://doi.org/10.3168/jds.2014-8576

King, M. T. M., S. J. LeBlanc, E. A. Pajor, T. C. Wright, and T. J. DeVries, 2018. Behavior and productivity of cows milked in automated systems before diagnosis of health disorders in early lactation. J. Dairy Sci. 101:4343-4356. https://doi.org/10.3168/jds.2017-13686

Kirby, K. N. and D. Gerlanc, 2013. BootES: An R package for bootstrap confidence intervals on effect sizes. Behav. Res. 45:905-927. https://doi.org/10.3758/s13428-013-0330-5

Kováč, G., C. Tóthová, O. Nagy, O., and H. Seidel, 2011. Milk amyloid A and selected serum proteins in cows suffering from mastitis. Acta Vet. Brno 80:3-9.

https://doi.org/10.2754/avb201180010003

Kovačević-Filipović, M., V. Ilić, Z. Vujčić, B. Dojnov, M. Stevanov-Pavlović, Z. Mijačević, and T. Božić, 2012. Serum amyloid A isoforms in serum and milk from cows with Staphylococcus aureus subclinical mastitis. Vet. Immunol. Immunopathol. 145(1-2):120-128. https://doi.org/10.1016/j.vetimm.2011.10.015

Kume, S., K. Nonaka, T. Oshita, and T. Kozaka, 2010. Evaluation of drinking water intake, feed water intake and total water intake in dry and lactating cows fed silages. Livest. Sci. 128:46-51. https://doi.org/10.1016/j.livsci.2009.10.012

Kyriazakis, I. and B. J. Tolkamp, 2011. Hunger and Thirst. Pages 44-57 in Animal Welfare, Appleby, M.C., J. A. Mench, I. A. S. Olsson, and B. O. Hughes, Eds. 2nd ed. CAB International, Wallingford, UK. 
Lecchi, C., F. Dilda, P. Sartorelli, and F. Ceciliani, 2012. Widespread expression of SAA and Hp RNA in bovine tissues after evaluation of suitable reference genes. Vet. Immunol. Immunopathol. 145:556-562. https://doi.org/10.1016/j.vetimm.2011.12.017

Lee, D. K., 2016. Alternatives to P value: confidence interval and effect size. Korean J. Anesthesiol. 69(6):555-562. https://doi.org/10.4097/kjae.2016.69.6.555

Littin, K., A. Acevedo, W. Browne, J. Edgar, M. Mendl, D. Owen, C. Sherwin, H. Würbel, and C. J. Nicol, 2008. Towards humane end points: Behavioural changes precede clinical signs of disease in a Huntington's disease model. Proc. Biol. Sci. 275:1865-1874. https://doi.org/10.1098/rspb.2008.0388

Lomb, J., H. W. Neave, D. M. Weary, S. J. LeBlanc, J. M. Huzzey, and M. A. G. von Keyserlingk, 2018. Changes in feeding, social, and lying behaviors in dairy cows with metritis following treatment with a nonsteroidal anti-inflammatory drug as adjunctive treatment to an antimicrobial. J. Dairy Sci. 101(5):4400-4411.

https://doi.org/10.3168/jds.2017-13812

Lukas, J. M., J. K. Reneau, and J. G. Linn, 2008. Water intake and dry matter intake changes as a feeding management tool and indicator of health and estrus status in dairy cows. J. Dairy Sci. 91(9):3385-3394. https://doi.org/10.3168/jds.2007-0926

Mandel, R., H. R. Whay, C. J. Nicol, and E. Klement, 2013. The effect of food location, heat load, and intrusive medical procedures on brushing activity in dairy cows. J. Dairy Sci. 96:6506-6513. https://doi.org/10.3168/jds.2013-6941

Mandel, R., C. J. Nicol, H. R. Whay, and E. Klement, 2017. Short communication: Detection and monitoring of metritis in dairy cows using an automated grooming device. J. Dairy Sci. 100:5724-5728. https://doi.org/10.3168/jds.2016-12201

Manson, F. J. and M. C. Appleby, 1990. Spacing of dairy cows at a food trough. Appl. Anim. Behav. Sci. 26:69-81. https://doi.org/10.1016/0168-1591(90)90088-U

Madouasse, A., J. N. Huxley, W. J. Browne, A. J. Bradley, and M. J. Green, 2010. Somatic cell count dynamics in a large sample of dairy herds in England and Wales. Prev. Vet. Med. 96:56-64. https://doi.org/10.1016/j.prevetmed.2010.05.005

McConnachie, E., A. M. C. Smid, A. J. Thompson, D. M. Weary, M. A. Gaworski, and M. A. G. Von Keyserlingk, 2018. Cows are highly motivated to access a grooming substrate. Biol. Lett. 14:1-4. https://doi.org/10.1098/rsbl.2018.0303

Medrano-Galarza, C., J. Gibbons, S. Wagner, A. M. de Passillé, and J. Rushen, 2012. Behavioral changes in dairy cows with mastitis, J. Dairy Sci. 95(12):6994-7002. https://doi.org/10.3168/jds.2011-5247

Munksgaard, L., M. B. Jensen, L. J. Pedersen, S. W. Hansen, and L. Matthews, 2005. Quantifying behavioural priorities - Effects of time constraints on behaviour of dairy cows, Bos taurus. Appl. Anim. Behav. Sci. 92:3-14. https://doi.org/10.1016/j.applanim.2004.11.005 
1118 Murata, H., N. Shimada, and M. Yoshioka, 2004. Current research on acute phase proteins in

1119 veterinary diagnosis: An overview. Vet. J. 168:28-40. https://doi.org/10.1016/s10900233(03)00119-9

Nakagawa, S., 2004. A farewell to Bonferroni: the problems of low statistical power and publication bias. Behav. Ecol. 15(6):1044-1045. https://doi.org/10.1093/beheco/arh107

Nakagawa, S. and I. C. Cuthill, 2007. Effect size, confidence interval and statistical significance: a practical guide for biologists. Biol. Rev. 82:591-605.

https://doi.org/10.1111/j.1469-185x.2007.00027.x

Nakagawa, S. and T. M. Foster, 2004. The case against retrospective statistical power analyses with an introduction to power analysis, Acta Ethol. 7:103-108.

https://doi.org/10.1007/s10211-004-0095-z

Napolitano, F., U. Knierim, F. Grasso, and G. De Rosa, 2009. Positive indicators of cattle welfare and their applicability to on-farm protocols. Ital. J. Anim. Sci. 8 (s1):355-365. https://doi.org/10.4081/ijas.2009.s1.355

Neave H. W., J. Lomb, D. M. Weary, S. J. Leblanc, J. M. Huzzey, and M. A. G. Von Keyserlingk, 2018. Behavioral changes before metritis diagnosis in dairy cows. J. Dairy Sci. 101(5):4388-4399. https://doi.org/10.3168/jds.2017-13078

O’Mahony, M. C., A. M. Healy, D. Harte, K. G. Walshe, P. R. Torgerson, and M. L. Doherty, 2006. Milk amyloid A: Correlation with cellular indices of mammary inflammation in cows with normal and raised serum amyloid A. Res. Vet. Sci. 80:155-161. https://doi.org/10.1016/j.rvsc.2005.05.005

Opp, M. R., 2005. Cytokines and sleep. Sleep Med. Rev. 9(5):355-364. https://doi.org/10.1016/j.smrv.2005.01.002

Patbandha, T. K., T. K. Mohanty, S. S. Layek, A. Kumaresan, and K. Behera, 2012. Application of pre-partum feeding and social behaviour in predicting risk of developing metritis in cross bred cows. Appl. Anim. Behav. Sci. 139:10-17.

https://doi.org/10.1016/j.applanim.2012.03.014

Petersson-Wolfe, C.S., Leslie, K.E. and Swartz, T.H., 2018. An update on the effect of clinical mastitis on the welfare of dairy cows and potential therapies. Veterinary Clinics: Food Animal Practice, 34(3), pp.525-535.

Pyörälä, S., M. Hovinen, H. Simojoki, J. Fitzpatrick, P. D. Eckersall, and T. Orro, 2011. Acute phase proteins in milk in naturally acquired bovine mastitis caused by different pathogens. Vet. Rec. 168(20):535. https://doi.org/10.1136/vr.d1120

Rahman, M., U. Müller, H. Sauerwein, P. Heimberg, and F. Ceciliani, 2013. Investigation of salivary acute phase proteins in calves. Pages 213-216 in Farm Animal Proteomics 2013, de Almeida A. et al. (eds). Wageningen Academic Publishers, Wageningen. 
1166 Sato, S., S. Sako, and A. Maeda, 1991. Social licking patterns in cattle (Bos taurus): influence of environmental and social factors. Appl. Anim. Behav. Sci. 32:3-12. https://doi.org/10.1016/S0168-1591(05)80158-3

Sato, S., K. Tarumizu, and K. Hatae, 1993. The influence of social factors on allogrooming in cows. Appl. Anim. Behav. Sci. 38:235-244. https://doi.org/10.1016/0168-1591(93)90022-H

Schirmann, K., D. M. Weary, W. Heuwieser, N. Chapinal, R. L. A. Cerri, and M. A. G. von Keyserlingk, 2016. Short communication: Rumination and feeding behaviors differ between healthy and sick dairy cows during the transition period. J. Dairy Sci. 99:9917-9924. https://doi.org/10.3168/jds.2015-10548

Sepúlveda-Varas, P., D. M. Weary, and M. A. G. von Keyserlingk, 2014. Lying behavior and postpartum health status in grazing dairy cows. J. Dairy Sci. 97:6334-6343.

https://doi.org/10.3168/jds.2014-8357

Sepúlveda-Varasa, P., K. L. Proudfoot, D. M. Weary, and M. A. G. Keyserlingk, 2016. Changes in behaviour of dairy cows with clinical mastitis. Appl. Anim. Behav. Sci. 175:8-13. https://doi.org/10.1016/j.applanim.2014.09.022

Siivonen, J., S. Taponen, M. Hovinen, M. Pastell, B. Joop Lensink, S. Pyörälä, and L. Hanninen, 2011. Impact of acute clinical mastitis on cow behaviour. Appl. Anim. Behav. Sci. 132:101-106. https://doi.org/10.1016/j.applanim.2011.04.005

Sordillo, L. M., K. Shafer-Weaver, and D. DeRosa, 1997. Immunobiology of the mammary gland. J. Dairy Sci. 80:1851-1865. https://doi.org/10.3168/jds.S0022-0302(97)76121-6

Stangaferro, M. L., R. Wijma, L. S. Caixeta, M. A. Al-Abri, and J. O. Giordano, 2016. Use of rumination and activity monitoring for the identification of dairy cows with health disorders. Part II. Mastitis. J. Dairy Sci. 99:7411=7421. https://doi.org/10.3168/jds.2016-10907

Steensels, M., E. Maltz, C. Bahr, D. Berckmans, A. Antler, and I. Halachmi, 2017. Towards practical application of sensors for monitoring animal health: the effect of post-calving health problems on rumination duration, activity and milk yield. J. Dairy Res. 84:132-138. https://doi.org/10.1017/S0022029917000176

Ternman, E., L. Hänninen, M. Pastell, S. Agenäs, and P. Nielsen, 2013. Sleep in dairy cows recorded with a non-invasive EEG technique. Appl. Anim. Behav. Sci. 140:25-32. https://doi.org/10.1016/j.applanim.2012.05.005

Toaff-Rosenstein, R. L., L. J. Gershwin, A. J. Zanella, and C. B. Tucker, 2016. The sickness response in steers with induced bovine respiratory disease before and after treatment with a non-steroidal anti-inflammatory drug. Appl. Anim. Behav. Sci. 181:49-62.

https://doi.org/10.1016/j.applanim.2016.05.016

Val-Laillet, D., A. M. de Passille, J. Rushen, and M. A. G. von Keyserlingk, 2008. The concept of social dominance and the social distribution of feeding-related displacements between cows. Appl. Anim. Behav. Sci. 111:158-172.

https://doi.org/10.1016/j.applanim.2007.06.001 
Page 39 of 4ioRxiv preprint doi: https://doi.org/10.1101/2021.01.10.426092; this version.posted April 22, 2021. The copyright holder for this preprint

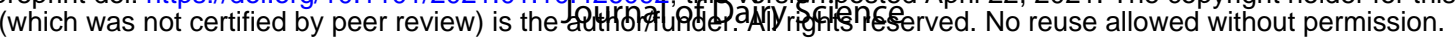

1217 Veissier I., M. M. Mialon, and K. H. Sloth, 2017. Short communication: Early modification

1218 of the circadian organization of cow activity in relation to disease or estrus. J. Dairy Sci.

1219 100:3969-3974. https://doi.org/10.3168/jds.2016-11853

1220

1221

1222

1223

1224

1225

1226

1227

1228

1229

1230

1231

1232

1233

1234

1235

1236

1237

1238

1239

1240

1241

1242

Verbeke, J., S. Piepers, K. Supré, and S. De Vliegher, 2014. Pathogen-specific incidence rate of clinical mastitis in Flemish dairy herds, severity, and association with herd hygiene. J. Dairy Sci. 97:6926-6934. https://doi.org/10.3168/jds.2016-11853

von Keyserlingk, M.A.G. and D. M. Weary, 2010. Review: Feeding behaviour of dairy cattle: Measures and applications. Can. J. Anim. Sci. 90(3):303-309.

https://doi.org/10.4141/CJAS09127

Wagner, N., V. Antoine, M. M. Mialon, R. Lardy, M. Silberberg, J. Koko, and I. Veissier, 2020. Machine learning to detect behavioural anomalies in dairy cows under subacute ruminal acidosis. Comput. Electron. Agric. 170:105233.

https://doi.org/10.1016/j.compag.2020.105233

Weary, D. M., J. M. Huzzey, and M. A. G. von Keyserlingk, 2009. Using behavior to predict and identify ill health in animals. J. Anim. Sci. 87:770-777. https://doi.org/10.2527/jas.20081297

Weigele, H. C., L. Gygax, A. Steiner, B. Wechsler, and J. B. Burla, 2017. Moderate lameness leads to marked behavioral changes in dairy cows. J. Dairy Sci. 101:2370-2382.

https://doi.org/10.3168/jds.2017-13120

Yeiser, E. E., K. E. Leslie, M. L. McGilliard, and C. S. Petersson-Wolfe, 2012. The effects of experimentally induced Escherichia coli mastitis and flunixin meglumine administration on activity measures, feed intake, and milk parameters. J. Dairy Sci. 95:4939-4949.

https://doi.org/10.3168/jds.2011-5064

1247

1248

1249

1250

1251

1252

1253

1254

1255

1256

1257

1258

1259

1260

1261

1262

1263

1264 
1265 Figure 1: Plan of the home pen including CCTV camera position and virtual division of floor-space 1266 (F1-13, M1/7/13, B1-13) for logging cow position. SB = salt bin, T1-3 = water troughs, B = rotating 1267 brush

1268

CCTV2

CCTV

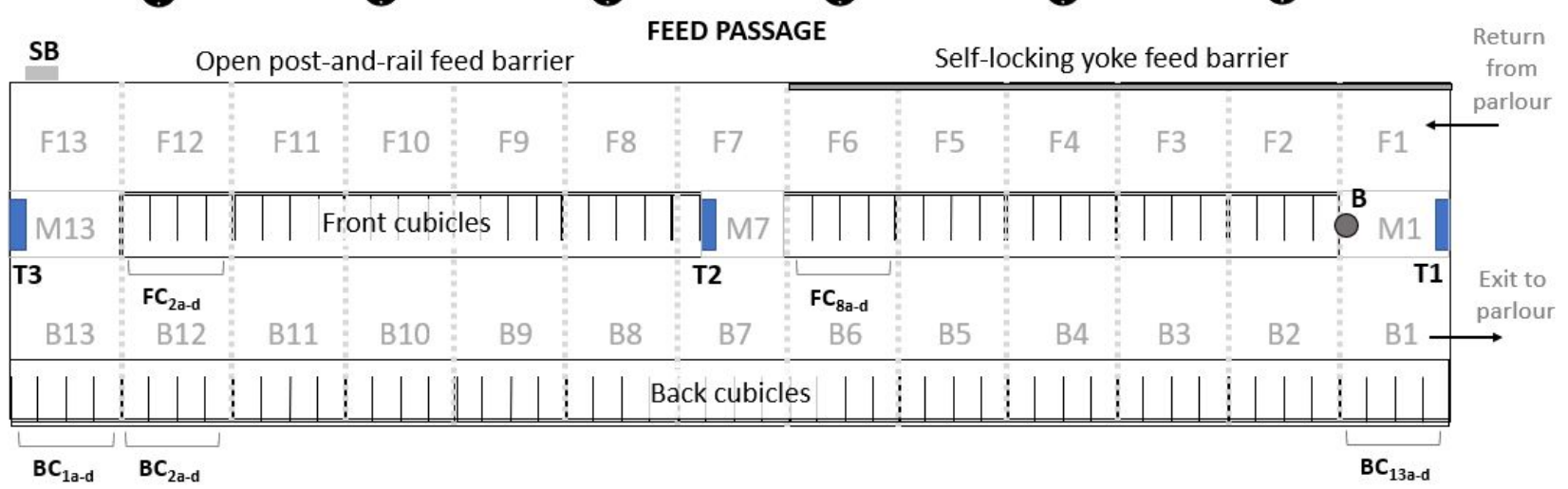

1271

1272

1273

1274

1275

1276

1277

1278

1279

1280

1281

1282

1283

1284

1285

1286

1287

1288

1289

1290 
Figure 2: The significant positive relationship between somatic cell count (SCC) in milk and salivary

1295 serum amylase-A (SAA) in dairy cattle $\left(\mathrm{R}^{2}=0.233 ; \mathrm{y}=31.715 \mathrm{x}^{0.4815}\right)$

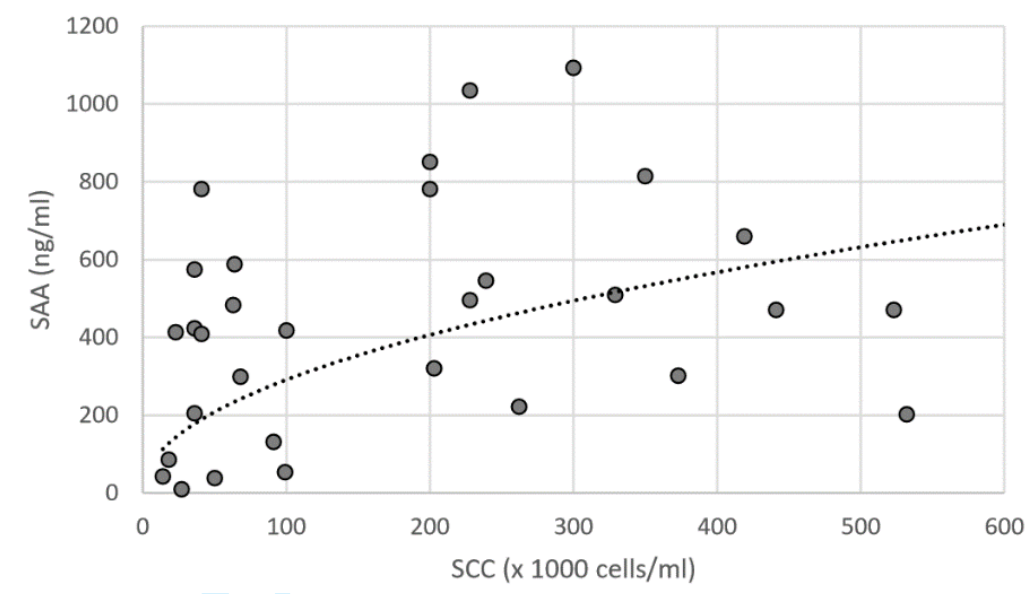


1331 Figure 3: Quadratic relationship between somatic cell count (SCC) and two measures of activity in 1332 cows with ( $\mathrm{SCM}=$ black), and without (CTRL = open circle) subclinical mastitis: (a) behavioral 1333 transitions ('Trans'); (b) distance covered ('Dist').

1334 (a)

1335

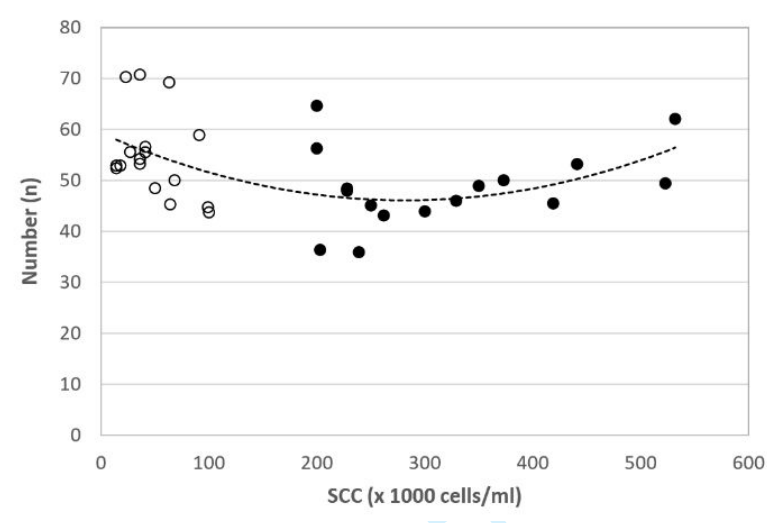

(b)

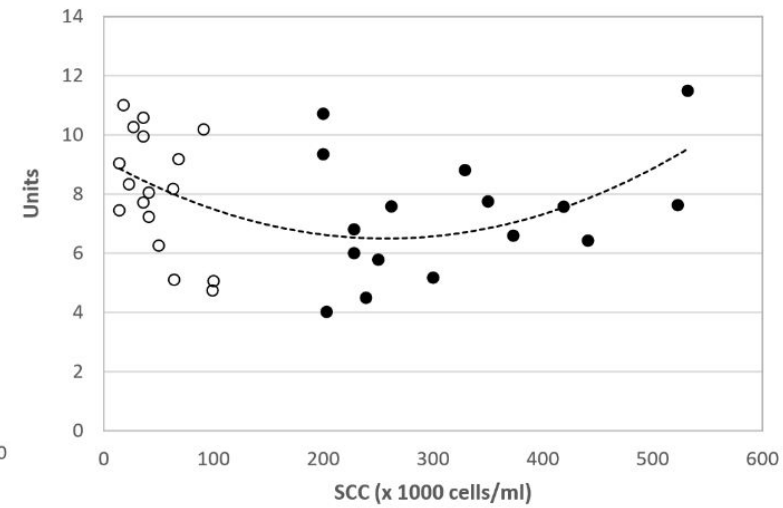




\section{The STROBE-Vet statement checklist.}

\begin{tabular}{|c|c|c|c|}
\hline & Item & STROBE-Vet recommendation & Page \# \\
\hline \multirow[t]{2}{*}{$\begin{array}{l}\text { Title and } \\
\text { Abstract }\end{array}$} & 1 & $\begin{array}{l}\text { (a) Indicate that the study was an observational study and, if applicable, use a common } \\
\text { study design term }\end{array}$ & 1 \\
\hline & & $\begin{array}{l}\text { (b) Indicate why the study was conducted, the design, the results, the limitations, and the } \\
\text { relevance of the findings }\end{array}$ & 1 \\
\hline $\begin{array}{l}\text { Background / } \\
\text { rationale }\end{array}$ & 2 & Explain the scientific background and rationale for the investigation being reported & 2 \\
\hline \multirow[t]{2}{*}{ Objectives } & 3 & $\begin{array}{l}\text { (a) State specific objectives, including any primary or secondary prespecified hypotheses } \\
\text { or their absence }\end{array}$ & 4 \\
\hline & & (b) Ensure that the level of organization ${ }^{a}$ is clear for each objective and hypothesis & 4 \\
\hline Study design & 4 & Present key elements of study design early in the paper & 4 \\
\hline \multirow[t]{2}{*}{ Setting } & 5 & $\begin{array}{l}\text { (a) Describe the setting, locations, and relevant dates, including periods of recruitment, } \\
\text { exposure, follow-up, and data collection }\end{array}$ & 4 \\
\hline & & (b) If applicable, include information at each level of organization & $\mathrm{n} / \mathrm{a}$ \\
\hline \multirow[t]{4}{*}{ Participants ${ }^{b}$} & 6 & $\begin{array}{l}\text { (a) Describe the eligibility criteria for the owners/managers and for the animals, at each } \\
\text { relevant level of organization }\end{array}$ & 4 \\
\hline & & $\begin{array}{l}\text { (b) Describe the sources and methods of selection for the owners/managers and for the } \\
\text { animals, at each relevant level of organization }\end{array}$ & 4 \\
\hline & & (c) Describe the method of follow-up & $\mathrm{n} / \mathrm{a}$ \\
\hline & & $\begin{array}{l}\text { (d) For matched studies, describe matching criteria and the number of matched individuals } \\
\text { per subject (e.g., number of controls per case) }\end{array}$ & 5 \\
\hline \multirow[t]{3}{*}{ Variables } & 7 & $\begin{array}{l}\text { (a) Clearly define all outcomes, exposures, predictors, potential confounders, and effect } \\
\text { modifiers. If applicable, give diagnostic criteria }\end{array}$ & 5 \\
\hline & & (b) Describe the level of organization at which each variable was measured & 5 \\
\hline & & $\begin{array}{l}\text { (c) For hypothesis-driven studies, the putative causal-structure among variables should be } \\
\text { described (a diagram is strongly encouraged) }\end{array}$ & $2-4$ \\
\hline
\end{tabular}




\begin{tabular}{|c|c|c|c|}
\hline $\begin{array}{l}\text { Data sources / } \\
\text { measurement }\end{array}$ & $8^{*}$ & $\begin{array}{l}\text { (a) For each variable of interest, give sources of data and details of methods of } \\
\text { assessment (measurement). If applicable, describe comparability of assessment methods } \\
\text { among groups and over time }\end{array}$ & $5-10$ \\
\hline & & $\begin{array}{l}\text { (b) If a questionnaire was used to collect data, describe its development, validation, and } \\
\text { administration }\end{array}$ & $\mathrm{n} / \mathrm{a}$ \\
\hline & & $\begin{array}{l}\text { (c) Describe whether or not individuals involved in data collection were blinded, when } \\
\text { applicable }\end{array}$ & 5 \\
\hline & & $\begin{array}{l}\text { (d) Describe any efforts to assess the accuracy of the data (including methods used for } \\
\text { "data cleaning" in primary research, or methods used for validating secondary data) }\end{array}$ & $\mathrm{n} / \mathrm{a}$ \\
\hline Bias & 9 & $\begin{array}{l}\text { Describe any efforts to address potential sources of bias due to confounding, selection, or } \\
\text { information bias }\end{array}$ & $\mathrm{n} / \mathrm{a}$ \\
\hline \multirow[t]{3}{*}{ Study size } & 10 & (a) Describe how the study size was arrived at for each relevant level of organization & 30 \\
\hline & & $\begin{array}{l}\text { (b) Describe how non-independence of measurements was incorporated into sample-size } \\
\text { considerations, if applicable }\end{array}$ & $\mathrm{n} / \mathrm{a}$ \\
\hline & & $\begin{array}{l}\text { (c) If a formal sample-size calculation was used, describe the parameters, assumptions, } \\
\text { and methods that were used, including a justification for the effect size selected }\end{array}$ & $\mathrm{n} / \mathrm{a}$ \\
\hline $\begin{array}{l}\text { Quantitative } \\
\text { variables }\end{array}$ & 11 & $\begin{array}{l}\text { Explain how quantitative variables were handled in the analyses. If applicable, describe } \\
\text { which groupings were chosen, and why }\end{array}$ & $10-13$ \\
\hline \multirow[t]{5}{*}{$\begin{array}{l}\text { Statistical } \\
\text { methods }\end{array}$} & \multirow[t]{5}{*}{12} & $\begin{array}{l}\text { (a) Describe all statistical methods for each objective, at a level of detail sufficient for a } \\
\text { knowledgeable reader to replicate the methods. Include a description of the approaches to } \\
\text { variable selection, control of confounding, and methods used to control for non- } \\
\text { independence of observations }\end{array}$ & $10-13$ \\
\hline & & (b) Describe the rationale for examining subgroups and interactions and the methods used & $5-6$ \\
\hline & & (c) Explain how missing data were addressed & 10 \\
\hline & & $\begin{array}{l}\text { (d) If applicable, describe the analytical approach to loss to follow-up, matching, complex } \\
\text { sampling, and multiplicity of analyses }\end{array}$ & $\mathrm{n} / \mathrm{a}$ \\
\hline & & $\begin{array}{l}\text { (e) Describe any methods used to assess the robustness of the analyses (e.g., sensitivity } \\
\text { analyses or quantitative bias assessment) }\end{array}$ & $10-13$ \\
\hline Participants & $13^{*}$ & $\begin{array}{l}\text { (a) Report the numbers of owners/managers and animals at each stage of study and at } \\
\text { each relevant level of organization - e.g., numbers eligible, included in the study, } \\
\text { completing follow-up, and analyzed }\end{array}$ & 4 \\
\hline
\end{tabular}




\begin{tabular}{|c|c|c|c|}
\hline & & $\begin{array}{l}\text { (b) Give reasons for non-participation at each stage and at each relevant level of } \\
\text { organization }\end{array}$ & 10 \\
\hline & & (c) Consider use of a flow diagram and/or a diagram of the organizational structure & $\mathrm{n} / \mathrm{a}$ \\
\hline \multirow{3}{*}{$\begin{array}{l}\text { Descriptive data } \\
\text { on exposures } \\
\text { and potential } \\
\text { confounders }\end{array}$} & $14^{*}$ & $\begin{array}{l}\text { (a) Give characteristics of study participants (e.g., demographic, clinical, social) and } \\
\text { information on exposures and potential confounders by group and level of organization, if } \\
\text { applicable }\end{array}$ & 4 \\
\hline & & $\begin{array}{l}\text { (b) Indicate number of participants with missing data for each variable of interest and at all } \\
\text { relevant levels of organization }\end{array}$ & $14,15,16,18$ \\
\hline & & $\begin{array}{l}\text { (c) Summarize follow-up time (e.g., average and total amount), if appropriate to the study } \\
\text { design }\end{array}$ & $n / a$ \\
\hline \multirow[t]{3}{*}{ Outcome data } & $15^{*}$ & $\begin{array}{l}\text { (a) Report outcomes as appropriate for the study design and summarize at all relevant } \\
\text { levels of organization }\end{array}$ & $13-22$ \\
\hline & & (b) For proportions and rates, report the numerator and denominator & \\
\hline & & $\begin{array}{l}\text { (c) For continuous outcomes, report the number of observations and a measure of } \\
\text { variability }\end{array}$ & \\
\hline \multirow[t]{3}{*}{ Main results } & 16 & $\begin{array}{l}\text { (a) Give unadjusted estimates and, if applicable, adjusted estimates and their precision } \\
\text { (e.g., 95\% confidence interval). Make clear which confounders and interactions were } \\
\text { adjusted. Report all relevant parameters that were part of the model }\end{array}$ & $13-19$ \\
\hline & & (b) Report category boundaries when continuous variables were categorized & $n / a$ \\
\hline & & $\begin{array}{l}\text { (c) If relevant, consider translating estimates of relative risk into absolute risk for a } \\
\text { meaningful time period }\end{array}$ & $n / a$ \\
\hline Other analyses & 17 & $\begin{array}{l}\text { Report other analyses done,-such as sensitivity/robustness analysis and analysis of } \\
\text { subgroups }\end{array}$ & \\
\hline Key results & 18 & Summarize key results with reference to study objectives & $1,22,30-31$ \\
\hline $\begin{array}{l}\text { Strengths and } \\
\text { Limitations }\end{array}$ & 19 & $\begin{array}{l}\text { Discuss strengths and limitations of the study, taking into account sources of potential bias } \\
\text { or imprecision. Discuss both direction and magnitude of any potential bias }\end{array}$ & $30-31$ \\
\hline Interpretation & 20 & $\begin{array}{l}\text { Give a cautious overall interpretation of results considering objectives, limitations, } \\
\text { multiplicity of analyses, results from similar studies, and other relevant evidence }\end{array}$ & $30-31$ \\
\hline Generalizability & 21 & Discuss the generalizability (external validity) of the study results & 31 \\
\hline $\begin{array}{l}\text { Funding } \\
\text { Transparency }\end{array}$ & 22 & $\begin{array}{l}\text { (a) Funding- Give the source of funding and the role of the funders for the present study } \\
\text { and, if applicable, for the original study on which the present article is based } \\
\text { (b) Conflicts of interest-Describe any conflicts of interest, or lack thereof, for each author }\end{array}$ & 32 \\
\hline
\end{tabular}




\begin{tabular}{|l|l|l|}
\hline & $\begin{array}{l}\text { (c) Describe the authors' roles- Provision of an authors' declaration of transparency is } \\
\text { recommended } \\
\text { (d) Ethical approval- Include information on ethical approval for use of animal and human } \\
\text { subjects } \\
\text { (e) Quality standards-Describe any quality standards used in the conduct of the research }\end{array}$ \\
\hline
\end{tabular}

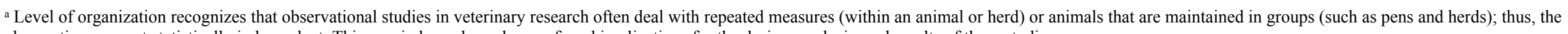
observations are not statistically independent. This non-independence has profound implications for the design, analysis, and results of these studies.

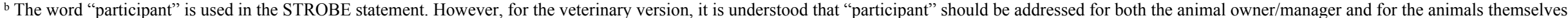

*Give such information separately for cases and controls in case-control studies and, if applicable, for exposed and unexposed groups in cohort and cross-sectional studies. 